\title{
数据驱动故障诊断方法泛化性能的经验性分析
}

\author{
郑怀亮 王日新 杨远涛 尹建程 徐敏强 \\ (哈尔滨工业大学深空探测基础研究中心 哈尔滨 150001)
}

\begin{abstract}
摘要: 近年来数据驱动的故障诊断方法被广泛研究, 但是这些方法有效的一个前提条件是训练诊断模型的数据与待测试数据 应需采集自相同的设备和运行环境, 然而这个前提条件在实际的诊断情形中很难得到满足, 实际能够用来训练诊断模型的通 常是采集自同类型设备或不同工况的历史数据。对于实际诊断情形下存在潜在差异的数据集，数据驱动故障诊断方法是否有 效的问题鲜有讨论。首先讨论了影响诊断方法泛化性能的可能因素, 然后构建了多个跨数据集诊断任务, 在此基础上对几个 数据驱动诊断方法的泛化性能进行了经验性的分析, 分析发现相较于模型复杂度数据集间的分布差异是影响跨域诊断泛化性 能的主要因素; 并进一步从信号特性分析的角度解释了设备型号差异和工况差异对跨域诊断性能影响的深层次原因。这些讨 论有益于启发面向实际诊断情形的数据驱动诊断方法的研究。
\end{abstract}

关键词: 故障诊断; 数据驱动; 泛化性能; 经验性分析

中图分类号: $\mathrm{TH} 17$

\section{An Empirical Analysis about the Generalization Performance of Data-driven Fault Diagnosis Methods}

\section{ZHENG Huailiang $\quad$ WANG Rixin YANG Yuantao YIN Jiancheng XU Minqiang}

(Deep Space Exploration Research Center, Harbin Institute of Technology, Harbin 150001)

\begin{abstract}
In recent years, data-driven fault diagnosis methods have been widely researched. A prerequisite of ensuring those methods' effectiveness is that the data for training diagnosis models and data to be tested should be collected from the same machine and the working environment. However, it is very difficult to satisfy this prerequisite in the actual diagnosis problem, and only the historical fault data collected from other same-type machines or different operating conditions are available for training the diagnosis model. The validity of conventional data-driven fault diagnosis methods for the actual diagnosis scenarios between datasets with potential discrepancy has rarely been discussed yet. The possible factors that dominate the generalization performance of fault diagnosis methods are first analyzed theoretically, and then multiple cross-dataset diagnosis tasks are organized. Based on them the empirical analysis about the generalization performance of several data-driven fault diagnosis methods is conducted. It is found that the distribution discrepancy between datasets is a major factor to influence the generalization performance. Meanwhile, the further fundamental reason for generalization performance declines is also explained from the perspective of signal characteristics under both model difference and operating condition difference. The discussion is conducive to inspiring the studies of data-driven diagnosis methods that can handle actual diagnosis scenarios.
\end{abstract}

Key words: fault diagnosis; data-driven; generalization performance; empirical analysis

\section{0 前言}

在现代工业中，机械系统的逐渐大型化、复杂 化、综合化和智能化对设备的状态监测和维修管理 提出了新的挑战。发展能够实施感知设备健康状态 及发展趋势的预测和健康管理(Prognostic and health

20190408 收到初稿, 20191121 收到修改稿 management, PHM)系统是保证设备运行可靠性、 安全性和经济性的重要手段 ${ }^{[1-2]}$ 。故障诊断是 PHM 系统中的重要环节, 是检测和识别系统故障模式的 重要技术。近年来, 随着人工智能技术的发展和工 业监测数据的积累, 数据驱动的故障诊断方法越来 越受到重视, 并且研究人员已经对该类型方法开展 了大量的研究工作 ${ }^{[3-5]}$ 。

数据驱动故障诊断方法的主要目的是希望从大量 的监测数据中学习故障的表现形式, 从而实现自主的 
故障模式识别。当前, 针对数据驱动故障诊断方法的 研究主要集中在特征提取和故障识别方法的研究两个 方面。在故障特征提取方面, 针对待诊断对象监测信 号的特点, 许多信号处理方法 ${ }^{[6-7]}$ 和信号动态特性描述 方法 ${ }^{[8-9]}$ 被应用和改进, 以期提取更加有辨识能力的故 障特征。在故障识别方法的构建方面, $k$ 近邻 $(k$-nearest neighbor, $\mathrm{KNN})^{[10]}$ 、人工神经网络 (Artificial neural network, $\mathrm{ANN})^{[11]}$ 以及支持向量机 (Support vector

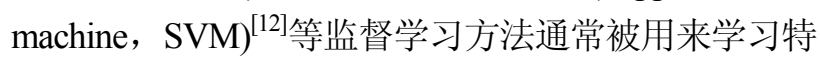
征向量与模式之间的映射关系。此外, 为了从原始监 测信号出发实现故障特征和识别模型的联合学习, 许 多深度学习技术也被应用于解决故障诊断问题 ${ }^{[13-15]}$ 。

虽然关于数据驱动故障诊断方法的研究已经取 得了一些成果, 但是当前的研究在验证方法有效性 时通常基于一个相同的前提设定, 即训练样本和测 试样本抽取自同一个原始的数据集 ${ }^{[10-13,16-20]}$ 。而这 个前提在实际诊断情形下非常难以满足, 通常能够 事先获得的训练数据可能是一些存在潜在差异的累 积故障数据, 例如同类型设备的实验室故障模拟实 验数据, 同类型设备的累积故障数据或者相同设备 但不同运行工况下的故障数据。从数据获取可行性 的角度出发, 跨数据集的诊断是实际诊断任务中更 加可能实现的情形。但是, 在这样的诊断情形下传 统数据驱动诊断方法是否有效以及可能影响其泛化 性能的主要因素等问题鲜有讨论。

本文的主要内容即是对传统数据驱动故障诊断 方法(主要是几个基于有监督学习的诊断方法)在跨 数据集诊断问题中的泛化性能进行经验性的分析。 具体而言, 本文首先从初步的理论分析角度明确了 几个可能影响诊断泛化性能的因素。然后, 通过组 织多个跨数据集诊断任务(包括齿轮和滚动轴承故 障诊断)经验的分析了多个数据驱动故障诊断方法 的泛化性能, 并分析了影响泛化性能的主要因素。 最后, 对导致该现象的深层次原因进行了探讨。通 过本文的分析可能启发和指导面向解决实际工程诊 断问题的数据驱动故障诊断方法的研究。

本文的组织结构为: 第 1 部分重点讨论了可能 影响诊断泛化性能的因素、研究跨数据集诊断的必 要性(实际诊断情形下的数据困境)以及明确本文要 研究的两个问题。第 2 部分主要介绍经验分析过程 中所利用的数据、所提取的诊断特征、以及泛化性 能和分布差异的度量方法; 第 3 部分主要对几个数 据驱动诊断方法的泛化性能进行讨论; 影响这些方 法泛化性能的主要因素即深层次原因将在第 4 部分 讨论; 最后, 在第 5 部分对该研究进行总结并对分 析过程中的不足进行讨论。

\section{1 问题的提出}

\section{1 影响诊断方法泛化性能的可能因素}

数据驱动故障诊断方法通常按图 1 所示的基本 流程来执行, 包括模型的训练和利用模型进行诊断 两个阶段。训练诊断模型阶段包含下述三个主要的 步骤 ${ }^{[13]}$ : (1) 数据的采集; (2) 特征提取和选择; (3) 识 别模型的构建。

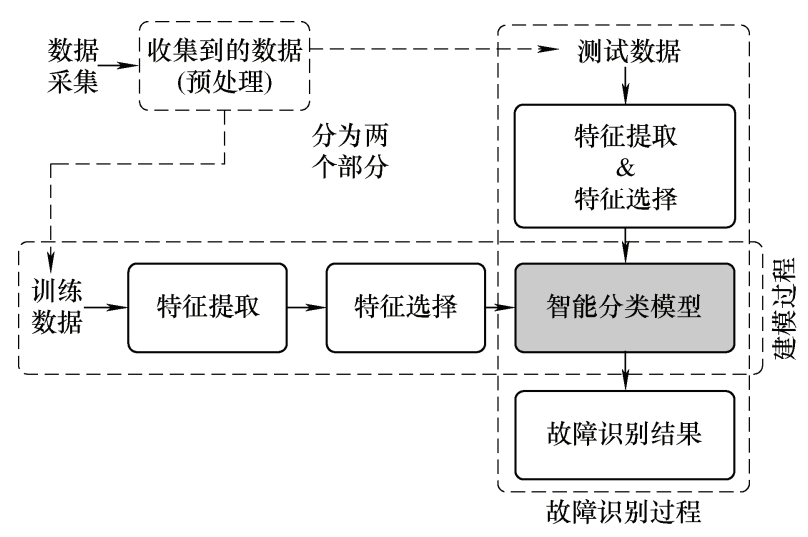

图 1 数据驱动故障诊断方法的基本流程

构建故障识别模型是依据经验历史数据确定特 征空间 $\mathcal{X}$ 到故障模式空间 $\mathcal{Y}$ 映射关系 $h: \mathcal{X} \rightarrow \mathcal{Y}$ 的 学习过程。诊断方法的泛化性能是指方法学习到的 模型对未知数据(即测试数据)的预测能力, 在统计 学习理论中通常利用模型对未知数据的泛化误差 $R(h)$ 来描述。在我们讨论的跨数据集情形下, 训练 数据和待测试数据可能抽取自不同的潜在分布, 不 妨设它们分别服从 $\mathcal{D}_{s}$ (训练数据)和 $\mathcal{D}_{t}$ (测试数据)。

Ben-David 等在文献[21] 中讨论了二类分类任务 $\mathcal{X} \rightarrow\{0,1\}$ 的泛化误差边界, 由下述定理给出。

定理 1(泛化误差边界): 设学习算法的假设空间 $\mathrm{H}$ 的 $\mathrm{VC}$-维为 $\mathrm{VC}(\mathrm{H})$, 训练样本集 $\left\{\left(\boldsymbol{x}_{i}, y_{i}\right)\right\}_{i=1}^{n}$ 从 $\mathcal{D}_{s}$ 独立同分布采样获得。则对于假设空间内的任意假 设 $h \in \mathrm{H}$, 至少以 $1-\delta$ 的概率下述不等式成立

$$
\begin{aligned}
R_{\mathrm{T}}(h) \leqslant & \hat{R}_{\mathrm{S}}(h)+\sqrt{\frac{4}{n}\left[\mathrm{VC}(\mathrm{H}) \lg \frac{2 e n}{\mathrm{VC}(\mathrm{H})}+\lg \frac{4}{\delta}\right]}+ \\
& d_{\mathfrak{H}}\left(\mathfrak{D}_{s}, \mathfrak{D}_{t}\right)+\lambda
\end{aligned}
$$

式中, $R_{\mathrm{S}}(h)=\mathbb{E}_{\boldsymbol{x} \sim \mathcal{D}_{s}}[|h(\boldsymbol{x})-y|]$ 为 $h$ 在训练集上的期 望风险; $\hat{R}_{\mathrm{S}}(h)$ 为其经验风险; $R_{\mathrm{T}}(h)=$ $\mathbb{E}_{\boldsymbol{x} \sim \mathcal{D}_{t}}[|h(\boldsymbol{x})-y|]$ 为 $h$ 在测试集上的期望风险; $d_{\mathcal{H}}\left(\mathcal{D}_{s}, \mathcal{D}_{t}\right)$ 是两个分布之间的距离度量 $(\mathcal{A}$ - distance); $\lambda$ 是一个小量; $e$ 是自然对数的底。

上述泛化误差边界的第(1)和第(2)项是同分布数 据下学习的泛化误差界, 它们与样本量 $n$ 以及学习 算法的复杂度(假设空间的容量 $\mathrm{VC}(\mathrm{H})$ ) 有关; 第(3) 
项是训练集和测试集分布的差异。上述定理表明, 实际的诊断过程中, 至少存在下述因素可能影响模 型在测试集上的泛化误差。

(1) 训练数据的质量和数量。

数据是诊断知识的载体, 是该类故障诊断方法 所依赖的基础, 因此用于训练模型的数据质量和数 量将是影响诊断模型泛化性能和诊断结果的一个因 素。具体而言, 训练数据的质量和数量包括下述几 个方面: 已标记样本数据量; 不同故障模式样本的 数量; 同一模式下数据的质量。

在数据驱动的故障诊断方法中, 推理故障特征

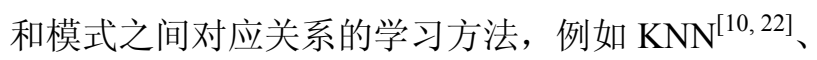
$\mathrm{ANN}^{[11,23]} 、 \mathrm{SVM}^{[12,24]}$ 、随机森林(Random forest, $R F)^{[25-26]}$ 以及自适应神经模糊推理系统 (Adaptive neuro-fuzzy inference system, ANFIS $)^{[27,28] \text { 等, 需要 }}$ 利用样本的标记信息。首先, 数据量是影响模型泛 化性能的重要因素, 如果数据量越大, 同样复杂度 的模型过拟合的可能性越小, 在测试集上的泛化能 力可能越高 ${ }^{[29]}$ 。因此, 已标记样本的数据量越多, 该类方法越可能正确识别测试样本。其次, 传统的 监督学习方法只能够识别训练集中已有的故障模 式, 当面临未见模式的新数据时不能对其中所蕴含 的知识进行识别。训练集中不同故障模式的数量是 所训练识别模型能够有效识别的模式数量上限。训 练集中包含的不同故障模式的数据越多, 识别模型 能够识别的模式越多。再者, 由传感器收集得到的 数据不可能是完美的, 实际测量数据可能受到测量 误差、噪声、传感器的精度和准确度等因素的影响; 此外, 还可能存在数据收集时由于人为因素导致的 离群点、遗漏和重复数据等 ${ }^{[30]}$ 。在同一故障模式下 收集的数据是否能够真实和全面地反映故障特性也 是影响故障诊断方法泛化性能的因素之一。

(2) 识别模型的复杂度。

从上述定理 1 中还发现, 模型的复杂度也是影 响泛化误差界的一个因素。模型的复杂度决定了识 别模型对训练数据的描述能力, 从这个角度来考虑, 如果模型过于简单则可能 “欠拟合” 训练数据, 使 得其对训练数据的经验风险很大(定理 1 中第(1)项), 模型的泛化性能将很低; 相反, 如果模型过于复杂 导致对训练数据 “过拟合”, 也将导致模型泛化误差 增加(定理 1 中的第(2项增加)。实际的学习过程是在 模型复杂度和对训练数据的经验风险之间寻找到一 个权衡, 使得整体泛化误差最小。此外增加训练数据 和特征降维也是降低过拟合风险的两个手段 ${ }^{[31]}$ 。

(3) 训练和测试数据的分布差异。

第三个可能的因素是训练集与测试集的分布差
异(定理 1 中的第(3)项)。通常, 诊断问题中设备的 状态是在由多个特征张成的空间内来描述的。特征 本身是用来反映信号在某一方面动态特性的定量化 参量, 基于对待诊断对象失效机理及其在监测信号 中可能表现形式的研究, 进行有针对性的特征提取 工作 ${ }^{[32]}$, 例如提取齿轮局部故障引起的振动冲击特 性来实现齿轮故障诊断。如果故障发生时恰好能够 改变监测信号在这一方面的特性, 则信号的该特征 参量可以被用来间接反映设备的健康状态。但是, 通常信号在某一方面的特性不单单受故障状态的影 响, 可能还会受到其他干扰因素的影响, 例如运行 工况、噪声、设备的结构差异、安装差异、制造误 差等。因此, 对于不同数据集来源的相同诊断问题 而言, 如果特征集合除了故障模式之外, 还对其他 因素敏感，那么可能导致训练集和测试集的分布之 间存在差异, 从而影响所训练的模型在测试集上的 泛化性能。

本文的第一个目的是分析在跨数据集情形下影 响诊断方法泛化性能的主要因素。重点对模型复杂 度和分布差异这两个因素进行充分分析。但是从理 论上计算一个特定学习算法的复杂度(比如 VC-维) 通常是非常困难的。因此, 我们将通过对多个不同 学习算法(模型复杂度不同)在大量跨数据集诊断任 务(分布差异不同)上的泛化能力进行经验性的分 析, 依据经验结果来探讨影响跨数据集诊断泛化性 能的主要因素。

\section{2 在实际诊断问题中的数据困境}

本小节主要讨论研究跨数据集故障诊断的必要 性问题。通过上面图 1 的执行过程发现，当前数据 驱动故障诊断方法暗含的一个前提条件是训练集和 测试集应采集自相同的设备、相同的工况条件、相 同的运行和测试环境、相同的传感器安装位置、甚 至相同的故障程度(即训练集和测试集从同一个原 数据集分裂获得)。但是面对实际工程中的诊断问 题, 按图 1 执行的数据驱动故障诊断方法面临的最 大的困难是在诊断之前很难获得该设备在当前运行 工况下大量的各类型故障样本。即这意味着需要在 诊断之前对待诊断设备进行各类型故障的模拟实验 来获取大量的训练数据。并且, 模拟实验中还需要 排除设备运行工况差异、设备运行和测试环境差异、 传感器安装位置差异以及故障程度差异等因素对测 试数据带来的一些影响。所以如果在利用这些数据 驱动诊断方法对每一个设备进行诊断之前都需要进 行有针对性的大量实验, 并且实验中还需要考虑所 有可能的影响因素, 这将是非常耗费资源甚至是难 以实现的。 
因此, 在实际的诊断问题中很难获得与待诊断 设备型号相同、工况相同、运行和测试环境相同、 传感器安装位置相同、甚至故障程度相同的历史训 练数据。我们称此为数据驱动诊断方法在实际诊断 问题中所面临的数据困境。

如前所述, 数据驱动故障诊断方法的目的是从 大量的历史数据中利用机器学习的方法学习故障特 征空间到故障模式空间的映射关系 ${ }^{[10,12-13,22-24,28]}$ 。 因此，历史数据中应该蕴含不同故障模式 “本质” 的表现形式。实际上, 在诊断一个设备之前有可能 获得采集自同设备但不同工况下运行时累积得到的 各故障模式数据、同型号但不同设备的累积故障数 据、该类型设备的实验室故障模拟数据等。

从获取数据的可行性角度出发, 上述来源的数 据才是训练数据驱动诊断模型可利用的数据, 但是 这些数据可能与一个(未来)待诊断设备产生的故障 数据存在潜在差异。从诊断知识获取的角度出发, 这些多来源的故障数据中应该蕴含着故障的本质特 性, 因为产生这些历史数据的设备与待诊断设备具 有相同的工作原理和相同的故障机理(只是工况或 型号不同)。因此, 利用机器学习方法从这些数据中 挖掘出特征和模式之间的对应关系, 并将这种关系 泛化到一个同类型设备的诊断问题中是解决数据驱 动诊断方法数据困境的可行途径。那么, 针对这种 跨不同来源数据集之间的诊断问题, 传统的数据驱 动故障诊断方法的诊断性能, 以及影响这些数据驱 动故障诊断方法性能的主要因素和深层次原因均值 得研究。

即本文重点讨论下述两个问题。

(1) 跨数据集诊断情形下, 影响数据驱动诊断 方法泛化性能的主要因素是什么? 本文主要通过经 验性分析的手段来讨论。

(2) 导致上述现象的深层次原因是什么? 即数据 集间的物理差异是如何影响到诊断方法泛化性能的。

在本文的研究中, 对训练数据的数量和质量等 因素不作讨论。

\section{2 预备工作}

\section{1 数据描述}

本文重点研究机械设备的故障诊断问题, 具体 讨论齿轮和滚动轴承两个部件, 它们是机械设备中 重要的传动和支撑部件, 是当前数据驱动故障诊断 方法研究中的重要应用对象。

(1) 齿轮数据。

PHM09 齿轮数据 ${ }^{[33]}$ 采集自 2 级传动齿轮箱, 在
齿轮箱的输入端和输出端分别布置一个加速度传感 器。小中间齿轮的故障实验包含正常、齿缺口和缺 齿三类健康状态。齿轮箱内部结构和故障齿轮见 图 2。实验中, 在各个健康状态下分别在输入轴的 转频为 $30 \mathrm{~Hz} 、 35 \mathrm{~Hz} 、 40 \mathrm{~Hz} 、 45 \mathrm{~Hz}$ 和 $50 \mathrm{~Hz}$, 以及高(High)、低(Low)两个负载下采集振动信号, 采样频率为 $66.6667 \mathrm{kHz}$ 。利用不同实验转速和载 荷下的数据来模拟存在差异的故障数据集, 这样在 齿轮诊断任务中共获得 10 个不同的数据集, 它们的 详细信息列于表 1 中。

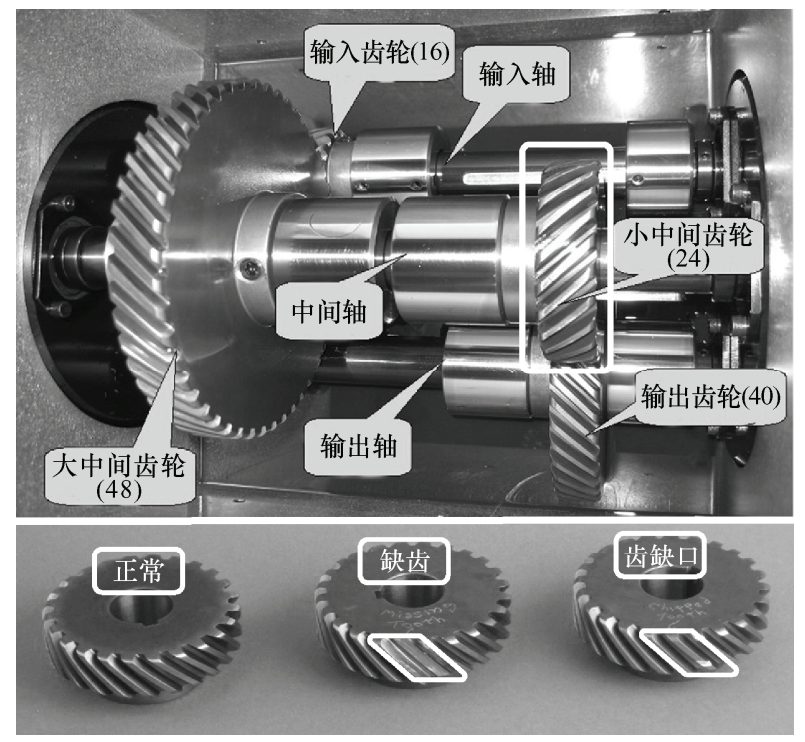

图 2 PHM09 齿轮箱内部结构和实验齿轮

表 1 PHM09 齿轮故障数据

\begin{tabular}{|c|c|c|c|c|}
\hline 数据集/Domain & 转频/Hz & 载荷 & 状态(标签) & 样本量 \\
\hline $30 \mathrm{~L} / 30 \mathrm{H}$ & 30 & $\mathrm{~L} / \mathrm{H}$ & $\begin{array}{lr}\text { 正常 } & (1) \\
\text { 齿缺口 } & (2) \\
\text { 缺齿 } & (3)\end{array}$ & $\begin{array}{l}100 \\
100 \\
100\end{array}$ \\
\hline $35 \mathrm{~L} / 35 \mathrm{H}$ & 35 & $\mathrm{~L} / \mathrm{H}$ & $\begin{array}{l}\text { 正常 (1) } \\
\text { 齿缺口 (2) } \\
\text { 缺齿 (3) }\end{array}$ & $\begin{array}{l}100 \\
100 \\
100\end{array}$ \\
\hline $40 \mathrm{~L} / 40 \mathrm{H}$ & 40 & $\mathrm{~L} / \mathrm{H}$ & 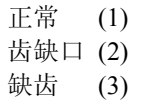 & $\begin{array}{l}100 \\
100 \\
100\end{array}$ \\
\hline $45 \mathrm{~L} / 45 \mathrm{H}$ & 45 & $\mathrm{~L} / \mathrm{H}$ & $\begin{array}{l}\text { 正常 (1) } \\
\text { 齿缺口 (2) } \\
\text { 缺齿 (3) }\end{array}$ & $\begin{array}{l}100 \\
100 \\
100\end{array}$ \\
\hline $50 \mathrm{~L} / 50 \mathrm{H}$ & 50 & $\mathrm{~L} / \mathrm{H}$ & $\begin{array}{ll}\text { 正常 } & (1) \\
\text { 齿缺口 } & (2) \\
\text { 缺齿 } & \text { (3) }\end{array}$ & $\begin{array}{l}100 \\
100 \\
100\end{array}$ \\
\hline
\end{tabular}

(2) 滚动轴承数据。

轴承数据共包含 11 个不同的域, 来自三个不同 的原始数据源: 凯斯西储大学(CWRU)轴承数据 ${ }^{[34]}$ 、 MFPT 轴承数据 ${ }^{[35]}$ 和一个全寿命试验数据。它们的 详细信息见表 2 。

利用 CWRU 数据组织了 8 个不同域 A-D 和 E-H。A-D 分别由驱动端轴承 (SKF 6205-2RS) 在 0-3 hp 四个不同运行载荷下的故障实验数据组成, 
每一个域共包含四个健康状态的数据: 正常状态 $\mathrm{N}$, 外环故障 $\mathrm{OR}$, 内环故障 IR 和滚动体故障 $\mathrm{B}$ 。每一 个故障状态包含三个不同故障程度 $(0.18 \mathrm{~mm}$, $0.36 \mathrm{~mm}$ 以及 $0.53 \mathrm{~mm}$ )的数据。与 A-D 的组织方 式相似, E-H 由风扇端轴承(SKF 6203-2RS)在 0-3 hp 四个不同运行载荷下的故障数据组成。 $\mathrm{A}-\mathrm{H}$ 的每一 个样本长度为 4000 , 采样频率为 $12 \mathrm{kHz}$ 。

表 2 滚动轴承故障数据

\begin{tabular}{|c|c|c|c|c|}
\hline 数据集/Domain & 轴承型号 & 工况 & 状态(标签) & 样本量 \\
\hline \multirow{4}{*}{$\begin{array}{l}\text { CWRU_DE_ } \\
\text { A-B-C-D }\end{array}$} & \multirow{4}{*}{6205} & O(1 $797 \mathrm{r} / \mathrm{min})-$ & $\mathrm{N}(1)$ & 270 \\
\hline & & $1(1772 \mathrm{r} / \mathrm{min})-$ & $\mathrm{OR}(2)$ & 270 \\
\hline & & $2(1750 \mathrm{r} / \mathrm{min})-$ & $\operatorname{IR}(3)$ & 270 \\
\hline & & $3(1730 \mathrm{r} / \mathrm{min})$ & B (4) & 270 \\
\hline \multirow{4}{*}{$\begin{array}{l}\text { CWRU_FE_ } \\
\text { E-F-G-H }\end{array}$} & \multirow{4}{*}{6203} & $0(1797 \mathrm{r} / \mathrm{min})-$ & $\mathrm{N}(1)$ & 270 \\
\hline & & $1(1772 \mathrm{r} / \mathrm{min})-$ & $\mathrm{OR}(2)$ & 270 \\
\hline & & 2(1 $750 \mathrm{r} / \mathrm{min})-$ & $\operatorname{IR}(3)$ & 270 \\
\hline & & $3(1730 \mathrm{r} / \mathrm{min})$ & B (4) & 270 \\
\hline \multirow{3}{*}{$\begin{array}{l}\mathrm{MFPT}_{-} \\
\text {I }\end{array}$} & \multirow{3}{*}{ 未知 } & $270 \mathrm{lbs}$ & $\mathrm{N}(1)$ & 100 \\
\hline & & $250 \sim 300 \mathrm{lbs}$ & $\mathrm{OR}(2)$ & 100 \\
\hline & & $250 / 300 \mathrm{lbs}$ & $\operatorname{IR}(3)$ & 100 \\
\hline $\mathrm{R}^{2} \mathrm{~F}_{-}$ & \multirow{2}{*}{6211} & $10.25 \mathrm{kN}$ & N (1) & 200 \\
\hline $\mathrm{J}$ & & (3 $000 \mathrm{r} / \mathrm{min})$ & $\operatorname{IR}(3)$ & 200 \\
\hline $\mathrm{R}^{2} \mathrm{~F}_{-}$ & \multirow{2}{*}{6211} & $10.25 \mathrm{kN}$ & $\mathrm{N}(1)$ & 200 \\
\hline K & & $(3000 \mathrm{r} / \mathrm{min})$ & $\operatorname{IR}(3)$ & 200 \\
\hline
\end{tabular}

利用 MFPT 数据组织了第 I 个数据集。该故障实 验的对象也是深沟球轴承, 轴承的详细几何参数为: 节径 $31.62 \mathrm{~mm}$, 滚动体直径 $5.97 \mathrm{~mm}$, 接触角 $0^{\circ}$, 滚动体个数 8 。该数据集包含三个健康状态: 正常状态 $\mathrm{N}$, 外环故障 $\mathrm{OR}$ 和内环故障 IR。内外环故障试验件如 图 3b 所示。试验中的轴转速为 $1500 \mathrm{r} / \mathrm{min}$, 每一个样 本的长度为 18000 , 采样频率为 $48828 \mathrm{~Hz}$ 。
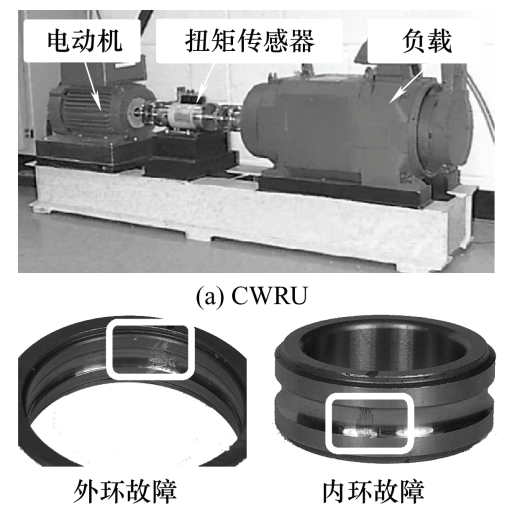

(b) MFPT

图 $3 \mathrm{CWRU}$ 试验台\&MFPT 故障试验件

此外, 还利用一个滚动轴承的全寿命试验数据 $(\mathrm{R} 2 \mathrm{~F})$ 组织了 $\mathrm{R} 2 \mathrm{~F} \_\mathrm{J}$ 和 $\mathrm{R} 2 \mathrm{~F} \_\mathrm{K}$ 两个数据集。该实验 的实验装置和最终的轴承故障形态见图 4 所示, 轴 承最终发生了内环故障。该试验的对象是 6211 深沟 球轴承: 节径 $77.50 \mathrm{~mm}$, 滚动体直径 $14.29 \mathrm{~mm}$,
接触角 $0^{\circ}$ 以及滚动体个数 10 。试验中轴承所受径向 载荷为 $10.25 \mathrm{kN}$, 轴的转速为 $3000 \mathrm{r} / \mathrm{min}$, 每间隔 $5 \mathrm{~min}$ 采集一次振动数据, 振动数据采样频率为 $24 \mathrm{kHz}$, 采样长度为 24000 个点。从全寿命数据中选 择三个片段(图 4), 片段 1 是轴承正常状态下的振动数 据，片段 2 是轴承早期内环故障状态下的数据，片段 3 是轴承严重内环故障状态下的数据。片段 1 和片段 2 组成 R2F_J, 片段 1 和片段 3 组成 R2F_K。

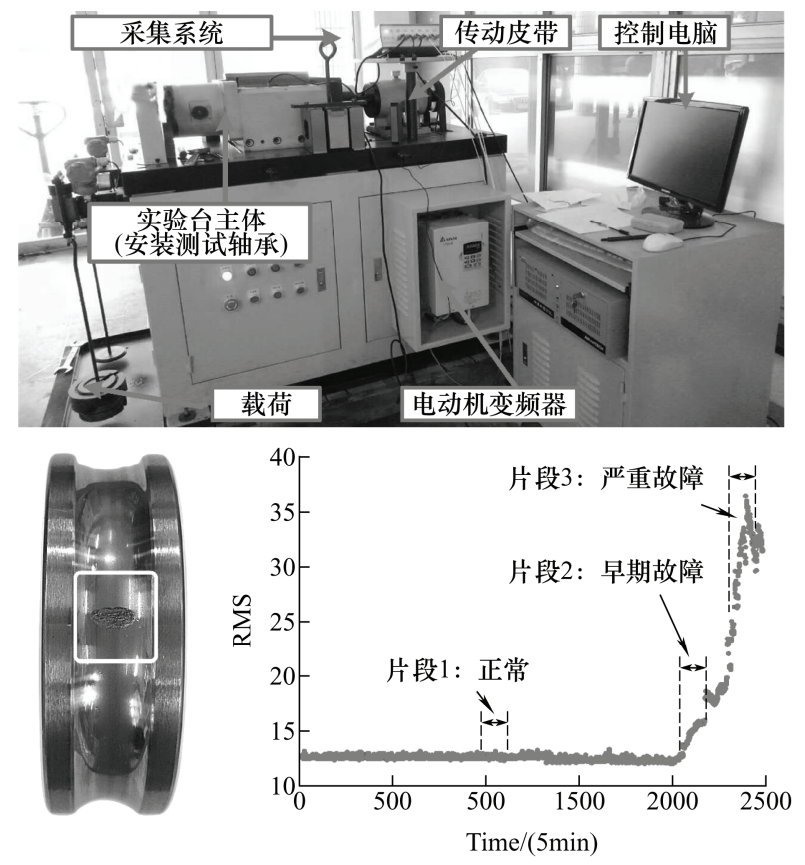

图 4 R2F 全寿命实验台和轴承试验结束时故障形态

\section{2 特征提取}

对于齿轮的故障诊断问题，分别从齿轮箱输 入端和输出端两个传感器采集的加速度信号中 提取 14 个特征, 形成 28 维特征向量以描述齿轮 的健康状态。这些特征分别是 6 个时域统计特征 参量(RMS, Kurtosis, Skewness, FM4, M6A, 和 Energy Ratio) ${ }^{[36]} ; 6$ 个频域统计特征参量 $(\mathrm{FC}$, RMSF, STDF, CP1, CP2, 和 CP 3$)^{[37]}$; 振动信 号幅值谱中的 1 阶转频幅值 AS- $1 \mathrm{x}$ 和 1 阶啮合频 率幅值 AS-m。

在滚动轴承故障诊断问题中，从各个数据集的 振动信号中提取 33 个特征来描述轴承的健康状态。 首先, 提取振动信号的 11 个时域统计特征参量 (Mean, std, SRA, RMS, Peak, Skewness, Kurtosis, CF, CLE, SF, IF)和 12 个频域统计特征参量 $(\mathrm{F} 1-\mathrm{F} 12)^{[38]}$ 。其次, 因为轴承故障缺陷引起的周期 性冲击会以周期性成分展现在振动信号的幅值谱 上, 或者以调制的形式展现在包络谱中 ${ }^{[39]}$, 因此从 振动信号的幅值谱和包络谱中分别提取了 1 阶转频 幅值和四个故障频率的幅值共 10 个参量(F-s, 
F-BPFI, F-BPFO, F-BFF, F-FTF; E-s, E-BPFI,

E-BPFO, E-BFF, E-FTF)。

\section{3 泛化性能和分布差异的度量}

将训练集和测试集分别表示为 $X_{s}=\left\{\left(\boldsymbol{x}_{i}, y_{i}\right)\right\}_{i=1}^{n}$ 和 $X_{t}=\left\{\left(\boldsymbol{x}_{i}, y_{i}\right)\right\}_{i=1}^{m}$, 其中 $x_{i} \in \mathbf{R}^{D}, y_{i} \in\{1,2, \cdots, C\}$; $D$ 为特征空间的维度, $C$ 为诊断问题中的模式个数。

(1) 泛化性能的度量。

故障诊断的泛化性能指的是一个故障模式识 别方法学习到的诊断模型对未知数据的预测能

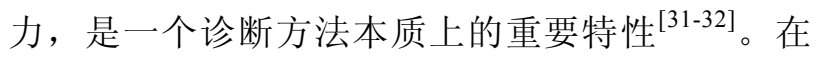
本文下述的讨论中, 将利用故障识别模型在测试 集上的识别精度 $A c c$ 来度量一个方法在测试集上 的泛化性能。识别精度 $A c c$ 的公式如下, 其中 $\tilde{y}_{i}$ 是 样本 $\boldsymbol{x}_{i}$ 的预测标签。

$$
A c c=\frac{\left|\boldsymbol{x}: \boldsymbol{x} \in X_{t} \cap \tilde{y}_{i}=y_{i}\right|}{\left|\boldsymbol{x}: \boldsymbol{x} \in X_{t}\right|}
$$

(2) 分布差异度量。

本文所涉及到的诊断任务中，训练集和测试集可 能来自于两个不同的概率分布 $\mathcal{D}_{s}$ 和 $\mathcal{D}_{t}$, 为了度量两个 分布之间的差异, 本文利用最大均值差异(Maximum mean discrepancy, MMD)来定量化的度量两个分布 之间的距离。 $\mathrm{MMD}$ 是一个非参数统计量, 它在再 生 Hilbert 空间(Reproducing kernel Hilbert space, RKHS) $\mathcal{H}$ 中度量两个分布的距离, 而不需要估计两 个分布的概率密度函数 ${ }^{[40]}$ 。对于训练集 $X_{S}$ 和测试集 $X_{t}$, 两个分布的经验 MMD 估计为

$$
\operatorname{MMD}_{\mathcal{H}}^{2}\left(X_{s}, X_{t}\right)=\left\|\frac{1}{n} \sum_{i=1}^{n} \phi\left(x_{i}\right)-\frac{1}{m} \sum_{j=1}^{m} \phi\left(x_{j}\right)\right\|_{\mathcal{H}}^{2}
$$

其中， $\phi: \mathcal{X} \mapsto \mathcal{H}$ 为原始空间 $\mathcal{X}$ 到 $\mathcal{H}$ 的映射。在故 障诊断问题中, 训练集和测试集之间均存在多种故 障模式的数据, 为了充分度量两个数据集间的差异, 我们基于 $\mathrm{MMD}$ 统计量构建了 $D_{m m d}$ 量来同时度量 两个数据集间的整体分布差异和各共有模式样本之 间的分布差异

$$
D_{m m d}=\operatorname{MMD}_{\mathcal{H}}\left(X_{s}, X_{t}\right)+\frac{1}{\mathrm{C}} \sum_{c=1}^{\mathrm{C}} \operatorname{MMD}_{\mathcal{H}}\left(X_{s}^{c}, X_{t}^{c}\right)
$$

其中

$$
\begin{gathered}
\operatorname{MMD}_{\mathcal{H}}\left(X_{s}, X_{t}\right)=\left\|\frac{1}{n} \sum_{i=1}^{n} \phi\left(\boldsymbol{x}_{i}\right)-\frac{1}{m} \sum_{j=1}^{m} \phi\left(\boldsymbol{x}_{j}\right)\right\|_{\mathcal{H}} \\
\operatorname{MMD}_{\mathcal{H}}\left(X_{s}^{c}, X_{t}^{c}\right)= \\
\left\|\frac{1}{n^{c}} \sum_{x_{i} \in X_{x}^{c}} \phi\left(\boldsymbol{x}_{i}\right)-\frac{1}{m^{c}} \sum_{\boldsymbol{x}_{j} \in X_{t}^{c}} \phi\left(\boldsymbol{x}_{j}\right)\right\|_{\mathcal{H}}
\end{gathered}
$$

$X_{s}^{c}=\left\{\boldsymbol{x}_{i}: \boldsymbol{x}_{i} \in X_{s} \wedge y_{i}=c\right\}$ 为训练集中 $c^{\text {th }}$ 类样本, $n^{c}=\left|X_{s}^{c}\right|$ 为样本量。 $X_{t}^{c}=\left\{\boldsymbol{x}_{j}: \boldsymbol{x}_{j} \in X_{t} \wedge y_{j}=c\right\}$ 为测 试集中的 $c^{\text {th }}$ 类样本, $m^{c}=\left|X_{t}^{c}\right|$ 为对应的样本量。

\section{3 数据驱动诊断方法在跨数据集} 诊断中的性能

本小节在固定的故障诊断特征集合和数据量之 下，重点展示多个不同的故障模式识别方法在多个 跨数据集诊断任务中的泛化性能。

\section{1 数据驱动的故障识别方法}

本文研究了下述 7 个有监督故障识别方法。

(1) SVM-RBF：支持向量机(RBF 核)。

(2) SVM-Linear: 支持向量机(Linear 核)。

(3) MLP: 多层感知机模型。

(4) LR: Logistic 回归模型。

(5) KNN: $k$ 近邻模型。

(6) DT: 决策树(CART)。

(7) RF: 随机森林。

这些方法在机械设备的故障诊断领域均有应用。 SVM 是基于结构风险最小化的学习方法, 它是定义在 特征空间上的间隔最大的线性分类模型 ${ }^{[29]}$ 。SVM-RBF 和 SVM-Linear 是分别利用 RBF 核 $k\left(\boldsymbol{x}_{i}, \boldsymbol{x}_{j}\right)=$ $\exp \left(-\frac{\left\|\boldsymbol{x}_{i}-\boldsymbol{x}_{j}\right\|^{2}}{2 \sigma^{2}}\right)$ 和 Linear 核 $k\left(\boldsymbol{x}_{i}, \boldsymbol{x}_{j}\right)=\boldsymbol{x}_{i}^{\mathrm{T}} \boldsymbol{x}_{j}$ 的 SVM 学习算法。MLP 是联结主义下的一个三层神 经网络模型, 利用 BP 算法训练网络参数 ${ }^{[31]}$ 。LR 是 统计学习中的经典分类方法 ${ }^{[31]}$, 也被应用于故障诊 断问题中。KNN 是基于模仿学习思想的经典算法 ${ }^{[41]}$ 。 DT 是基于符号化推理思想的基本分类方法，在我们 的实验中利用了经典的 CART 算法 ${ }^{[41]}$ 。 RF 是基于 决策树的集成学习算法 ${ }^{[42]}$ 。

\section{2 实验的执行细节}

在第 2.1 节中构建了多个关于齿轮诊断和滚 动轴承诊断的数据集, 利用这些数据集进一步可 以构建多个跨数据集的诊断任务。对于每一个诊 断任务, 均按照图 5a 中所示的流程来训练并测 试各个数据驱动故障识别方法。然后, 对原始特 征集合利用最小几余最大相关方法 (Minimal redundancy maximal relevance, mRMR $)^{[43]}$ 来进行 特征选择, 选择排序后的前 $[\mathrm{D} / 2]$ 个特征, 即齿轮 诊断任务选择 14 个特征, 滚动轴承诊断任务选 择 16 个特征。

各个诊断任务的识别结果将按照图 $5 \mathrm{~b}$ 所示的 方式展示, 图中的纵轴代表不同的训练集，横轴 代表不同的测试集，图中的对角线元素表示利用 数据集中的一部分训练模型然后识别剩余部分的实 


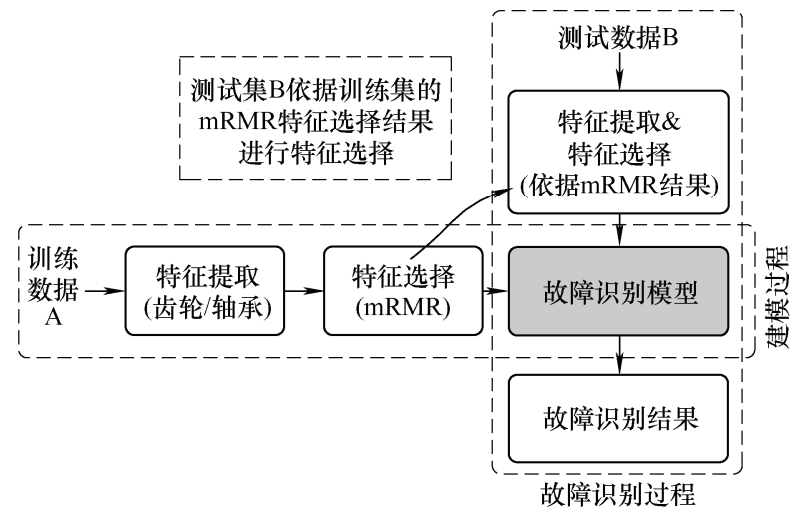

(a) 跨数据集诊断实验的执行过程

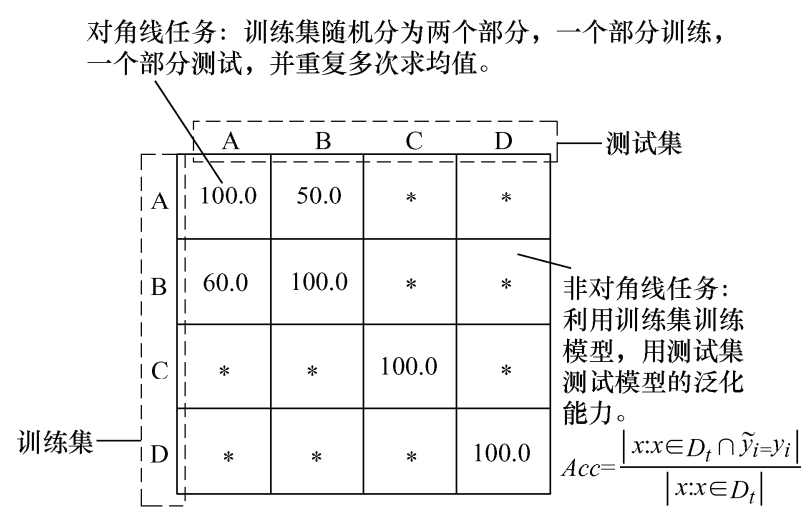

(b) 识别结果的展示方式

图 5 跨数据集诊断的执行流程及结果展示方式

验结果, 两个部分的划分比例为 $50 \%$, 并且进行 多次随机划分 (20 次) 以消除可能的随机影响。非 对角线元素代表两个不同来源数据集间的诊断 结果。

\section{3 识别结果}

各个故障识别方法在齿轮诊断任务和滚动轴承 诊断任务中的识别结果分别展示在图 6 和图 7 中。 从这些识别结果中可以发现以下几点。

识别精度 $A c c(\%)$ 齿轮诊断任务

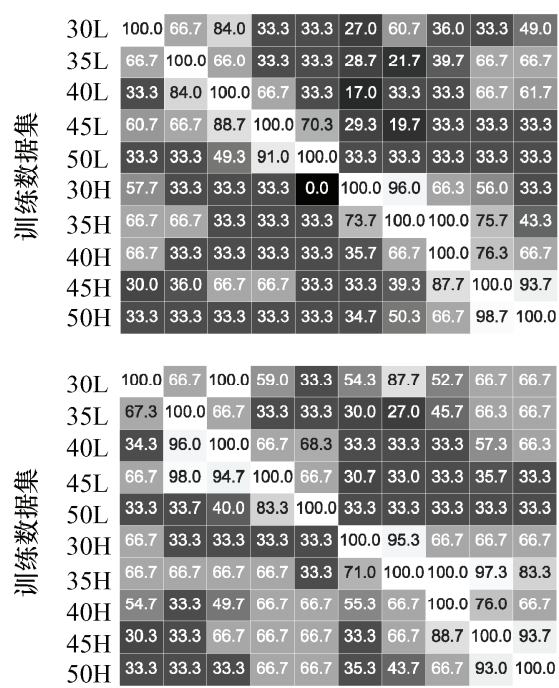

30L $100.088 .393 .7 \quad 66.762 .7 \quad 33.3 \quad 33.3 \quad 33.343 .756 .0$ 35L 79.7100 .069 .359 .748 .325 .353 .754 .356 .066 .7 40L $57.366 .7100 .066 .770 .7 \quad 7.0 \quad 25.032 .3 \quad 37.065$ 舞 45L $77.066 .7100 .0100 .082 .3 \quad 33.3 \quad 33.3 \quad 34.366 .767 .7$ 㱖 $50 \mathrm{~L} 52.066 .766 .766 .7100 .033 .357 .7 \quad 66.766 .766 .7$ $30 \mathrm{H} \quad 66.7 \quad 33.3 \quad 33.3 \quad 33.3 \quad 33.3 \quad 100.070 .766 .765 .3 \quad 33.3$ $35 \mathrm{H} 60.760 .760 .760 .760 .760 .7100 .068 .760 .760 .7$

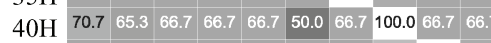
$45 \mathrm{H} 66.766 .066 .766 .766 .766 .766 .774 .3100 .068 .3$ $50 \mathrm{H} 66.766 .748 .366 .766 .751 .066 .766 .770 .3100 .0$

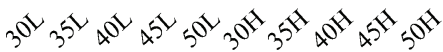
测试数据集

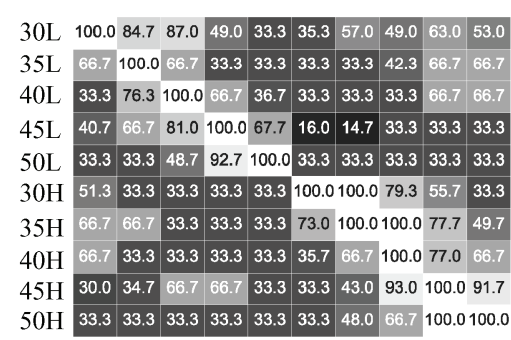

$\begin{array}{lllllllllll}30 \mathrm{~L} \quad 100.0 & 66.7 & 83.3 & 41.7 & 33.3 & 78.0 & 667 & 49.7 & 667 & 66.7\end{array}$ \begin{tabular}{lllllll|l|l|l|l}
$35 \mathrm{~L}$ & 66.7 & 100.0 & 66.7 & 33.3 & 33.3 & $\mathbf{2 . 3}$ & $\mathbf{1 2 . 7}$ & 40.7 & 66.3 & 66.7
\end{tabular} $\begin{array}{lllllllllllll}40 \mathrm{~L} & 33.3 & 77.7 & 100.0 & 66.7 & 66.7 & 33.3 & 33.3 & 33.3 & 66.7 & 65.7\end{array}$

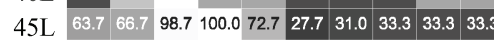
$\begin{array}{lllllllllllll}50 \mathrm{~L} & 33.3 & 33.3 & 66.7 & 92.0 & 100.0 & 33.3 & 33.3 & 33.3 & 33.3 & 33.3\end{array}$ $\begin{array}{lllllllllll}30 \mathrm{H} & 52.0 & 33.3 & 33.3 & 33.3 & 33.3 & 100.0 & 95.3 & 62.7 & 62.7 & 35.0\end{array}$ $35 \mathrm{H} \quad 66.7 \quad 33.3 \quad 33.3 \quad 33.3 \quad 33.3 \quad 76.0100 .0100 .092 .3 \quad 48.0$

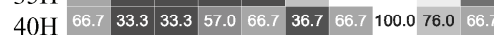
$\begin{array}{lllllllllll}45 \mathrm{H} & 32.3 & 62.7 & 66.7 & 66.7 & 36.7 & 33.3 & 43.3 & 80.0 & 100.0 & 89.0\end{array}$ $\begin{array}{llllllllll}50 \mathrm{H} & 33.3 & 33.3 & 33.3 & 33.3 & 66.7 & 40.0 & 60.3 & 66.7 & 100.0100 .0\end{array}$

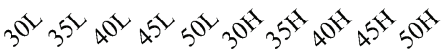
测试数据集

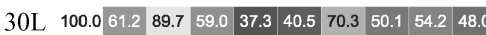
$\begin{array}{lllllllllll}35 \mathrm{~L} & 65.4 & 100.0 & 63.0 & 36.7 & 38.5 & 27.7 & 36.2 & 38.5 & 65.1 & 64.2\end{array}$

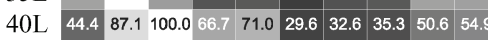
$\begin{array}{lllllllllll}45 \mathrm{~L} & 61.3 & 79.9 & 95.0 & 100.0 & 67.8 & 22.9 & 37.3 & 39.4 & 41.6 & 38.9\end{array}$ $\begin{array}{llllllllllll}50 \mathrm{~L} & 33.9 & 52.6 & 45.3 & 68.8 & 100.0 & 33.3 & 33.3 & 33.3 & 34.1 & 39.5\end{array}$ $\begin{array}{llllllllllll}30 \mathrm{H} & 55.6 & 35.2 & 44.9 & 47.4 & 40.6 & 100.0 & 95.4 & 68.4 & 66.7 & 62.7\end{array}$ $35 \mathrm{H} \quad 66.752 .766 .7 \quad 63.143 .3 \quad 71.5100 .0100 .091 .7 \quad 74.8$ $\begin{array}{lllllllllll}40 \mathrm{H} & 57.2 & 35.1 & 51.9 & 66.7 & 66.5 & 44.3 & 66.7 & 100.0 & 76.2 & 66.7\end{array}$ $45 \mathrm{H} \quad 19.437 .254 .666766034 .157 .5 \quad 87.11000942$

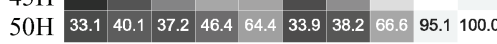

$\begin{array}{lllllllllllll}30 \mathrm{~L} & 100.0 & 33.3 & 33.3 & 33.3 & 33.3 & 33.3 & 33.3 & 32.3 & 58.7 & 38.7\end{array}$ $\begin{array}{lllllllllllll}35 \mathrm{~L} & 66.7 & 100.0 & 51.3 & 33.3 & 33.3 & 33.3 & 33.0 & 14.3 & 0.7 & 33.7\end{array}$ 40L $\begin{array}{llllllllllll}33.3 & 33.3 & 100.0 & 34.3 & 33.3 & 33.3 & 33.3 & 33.3 & 33.3 & 33.3\end{array}$ $\begin{array}{llllllllllll}45 \mathrm{~L} & 24.0 & 33.3 & 32.7 & 100.0 & 33.3 & 33.3 & 33.3 & 33.3 & 33.3 & 33.3\end{array}$ $\begin{array}{llllllllllll}50 \mathrm{~L} & 33.3 & 33.3 & 33.3 & 33.3 & 100.0 & 33.3 & 33.3 & 33.3 & 33.3 & 33.3\end{array}$ $\begin{array}{llllllllllll}30 \mathrm{H} & 33.3 & 33.3 & 33.3 & 33.3 & 33.0 & 100.0 & 64.0 & 88.7 & 78.0 & 40.3\end{array}$

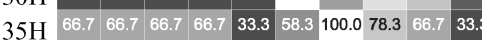

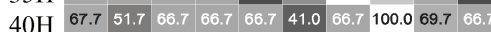
$\begin{array}{lllllllllll}45 \mathrm{H} & 66.3 & 66.7 & 89.7 & 67.0 & 97.3 & 36.3 & 66.7 & 75.3 & 99.8 & 68.0\end{array}$ $\begin{array}{lllllllllllll}50 \mathrm{H} & 31.3 & 66.7 & 33.3 & 33.3 & 33.3 & 33.3 & 33.3 & 33.3 & 36.7 & 99.5\end{array}$

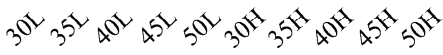
测试数据集

\begin{tabular}{|c|c|c|}
\hline 2 & 50 & 75 \\
\hline SVM_RBF & SVM_Linear & MLP \\
\hline LR & KNN & DT \\
\hline RF & & \\
\hline
\end{tabular}

图 6 各故障识别方法在齿轮跨数据集诊断任务中的识别精度

(1) 无论是在齿轮还是轴承诊断任务中, 当训 练集和测试集抽取自同一数据集时, 各个故障识别 方法均可以获得很高的泛化性能, 即图 6 和图 7 中 对角线上的识别精度很高。

(2) 对于齿轮的诊断任务, 各个识别方法在图 6 的非对角线任务(即不同工况下的数据集进行交叉
识别)中的泛化性能会降低。

(3) 对于滚动轴承诊断任务, 各个方法的泛化 性能在仅工况差异的非对角线任务中受到的影响较 小，但是在设备差异的非对角线任务中受到的影响 较大。

(4) 对于滚动轴承不同设备的非对角线诊断任 
务, 各个方法从 $\mathrm{E} / \mathrm{F} / \mathrm{G} / \mathrm{H} \rightarrow \mathrm{A} / \mathrm{B} / \mathrm{C} / \mathrm{D}$ 的性能要相对高 于从 $\mathrm{A} / \mathrm{B} / \mathrm{C} / \mathrm{D} \rightarrow \mathrm{E} / \mathrm{F} / \mathrm{G} / \mathrm{H}$ 的性能。

(5) 滚动轴承的诊断任务中, 当利用已有故 障数据集 $(\mathrm{A}-\mathrm{H})$ 训练识别模型去诊断一个实际的 内环失效轴承 $(\mathrm{R} 2 \mathrm{~F})$ 时, 各个方法的识别精度均 很低。
可见, 对于这些数据驱动故障诊断方法, 当训 练模型的训练集与测试集抽取自同一个数据集时均 可以获得较好的泛化性能。但是获得这样的数据在 实际工程中非常困难。如果利用采集自不同工况, 甚至其他同类型设备的数据集训练识别模型时, 这 些方法的泛化性能均会在不同程度上受到影响。

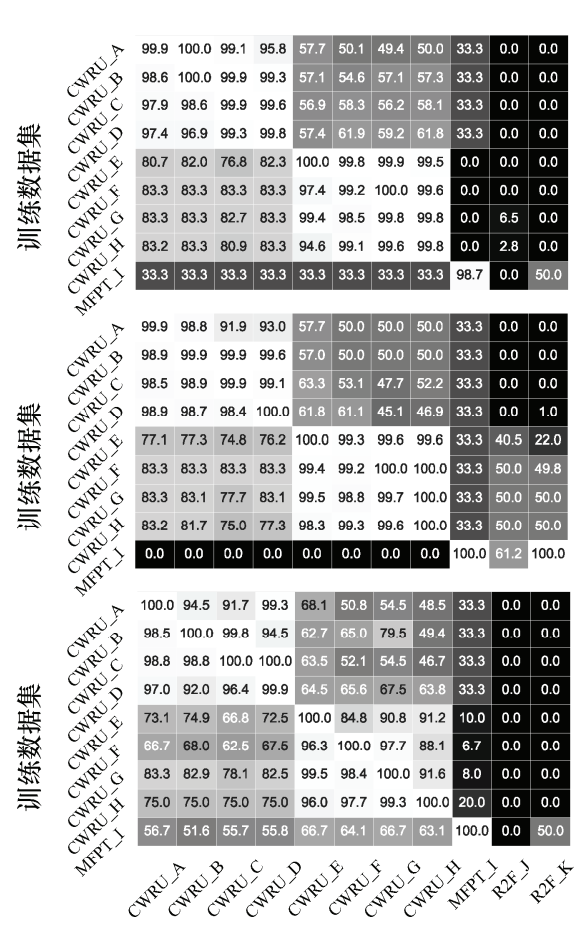

识别精度 $A c c(\%)$ _滚动轴承诊断任务

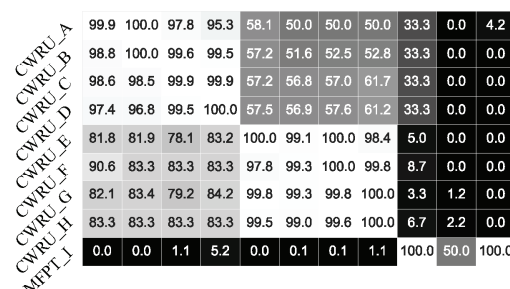

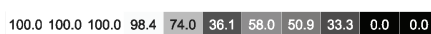

$\begin{array}{lllllllllll}99.3 & 100.0 & 99.9 & 99.7 & 73.5 & 72.2 & 77.4 & 62.4 & 33.3 & 0.0 & 0.0\end{array}$

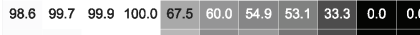

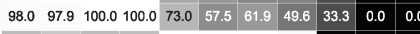

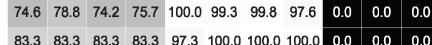

$\begin{array}{llllllllllll}77.6 & 83.1 & 83.3 & 83.3 & 97.3 & 100.0 & 1000 & 1000 & 0.0 & 0.0 & 0.0\end{array}$

$\begin{array}{llllllllllll}75.1 & 76.5 & 75.0 & 75.2 & 98.1 & 99.1 & 100.0 & 100.0 & 2.0 & 0.0 & 0.0\end{array}$

$\begin{array}{lllllllllllll}33.3 & 33.3 & 33.3 & 33.3 & 33.3 & 33.3 & 33.3 & 33.3 & 100.0 & 0.0 & 50.0\end{array}$

等

测试数据集

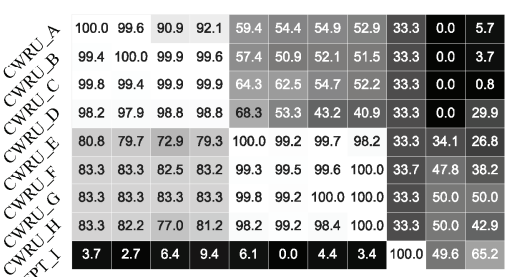

$\begin{array}{llllllllllll}99.8 & 91.9 & 91.7 & 91.3 & 68.9 & 44.8 & 50.9 & 49.2 & 33.3 & 50.0 & 0.0\end{array}$

$\begin{array}{llllllllllllll}99.4 & 99.0 & 99.1 & 98.4 & 85.9 & 64.4 & 86.7 & 53.2 & 26.7 & 45.5 & 0.0\end{array}$

$\begin{array}{lllllllllllll}98.1 & 97.8 & 99.8 & 98.2 & 78.7 & 45.1 & 56.7 & 50.0 & 33.3 & 50.0 & 0.0\end{array}$

$\begin{array}{llllllllllll}87.8 & 87.8 & 93.2 & 99.5 & 54.4 & 56.7 & 56.6 & 57.9 & 33.3 & 0.0 & 0.0\end{array}$

$\begin{array}{llllllllllll}80.6 & 81.5 & 75.0 & 76.9 & 99.9 & 84.3 & 90.1 & 90.9 & 10.0 & 0.0 & 0.0\end{array}$

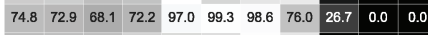

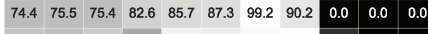

$\begin{array}{lllllllllllll}77.1 & 75.0 & 80.7 & 66.7 & 91.3 & 92.1 & 97.5 & 99.3 & 20.0 & 0.0 & 0.0\end{array}$

\begin{tabular}{|l|llllllllll|l|l|l|}
77.1 & 75.0 & 80.7 & 66.7 & 91.3 & 92.1 & 97.5 & 99.3 & 20.0 & 0.0 & 0.0 \\
\hline 44.4 & 44.4 & 44.4 & 44.4 & 66.7 & 65.6 & 65.3 & 66.7 & 99.6 & 32.5 & 60.5 \\
\hline
\end{tabular}

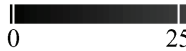

25

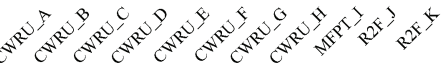

测试数据集
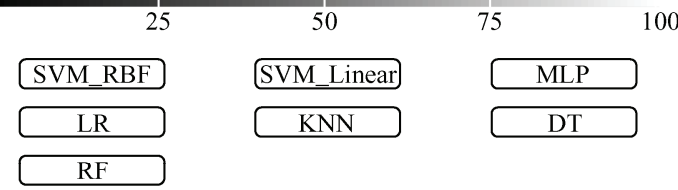

100

测试数据集

图 7 各故障识别方法在滚动轴承跨数据集诊断任务中的识别精度

\section{4 影响泛化性能的主要因素分析}

在下面的分析中，首先对影响跨数据集诊断泛 化性能的主要因素进行分析, 然后对导致该现象的 深层次原因做进一步探讨, 即从信号特性差异的角 度进行了进一步的解释。

\section{1 主要因素分析}

通过第 1 部分的分析明确了可能影响跨数据集 诊断泛化性能的三个因素: 数据量、模型复杂度和 分布差异。在本研究中不考虑数据量的影响, 主要 在数据量不变的情况下讨论模型复杂度和分布差异 这两个因素。

(1) 对模型复杂度的分析。

在数据量不变的情况下, 各个诊断方法在所有同 分布诊断任务中(图 6 和图 7 的对角线任务)均获得了 非常高的诊断性能。同时, 我们进一步在二维空间内 展示了各个故障识别方法的决策边界(决策边界与模 型复杂度有关)。这个试验与图 6 和图 7 对应试验的唯
一差别是在各个任务中只选择两个特征, 因此具体的 识别精度存在一些差异。图 8 中展示了齿轮诊断中 30L-30L 和 30L-45H 两个任务下各个方法的决策边 界。从图中发现, 虽然各个方法的决策边界不同, 但 是当训练集和测试集中的各类样本均非常聚集时, 只 要所构建的特征空间对各模式具有区分能力就可以 将从训练集学习到的决策模型泛化到目标测试集上 (如图 8 中的第一行所示)。但是, 当训练集和测试集 存在某些差异时, 仅要求特征空间具有模式区分能力 不足以实现泛化，因为此时在特征空间中两个数据集 中同类样本的位置可能存在很大差异。例如, 在图 8 的 $30 \mathrm{~L}-45 \mathrm{H}$ 任务中 $30 \mathrm{~L}$ 的三类样本与 $45 \mathrm{H}$ 的三类样 本在特征空间内并不相互重合，因此基于 $30 \mathrm{~L}$ 建立的 决策边界对 $45 \mathrm{H}$ 产生了错误的分类。同样, 图 9 中展 示了轴承诊断 CWRU_B-R2F_K 任务中各个方法的决 策边界。从图中同样发现两个数据集中的同类样本, 即正常类样本・和内环故障样本 $\boldsymbol{\Lambda}$, 也不互相聚集。 从而导致基于 CWRU_B 数据集训练得到的决策模型 不能正确识别 R2F_K 数据集。 
齿轮任务_2D决策边界: $1 \mathrm{st}$ 行 $30 \mathrm{~L} \rightarrow 30 \mathrm{~L}$ 任务; 2 nd行 $30 \mathrm{~L} \rightarrow 45 \mathrm{H}$ 任务
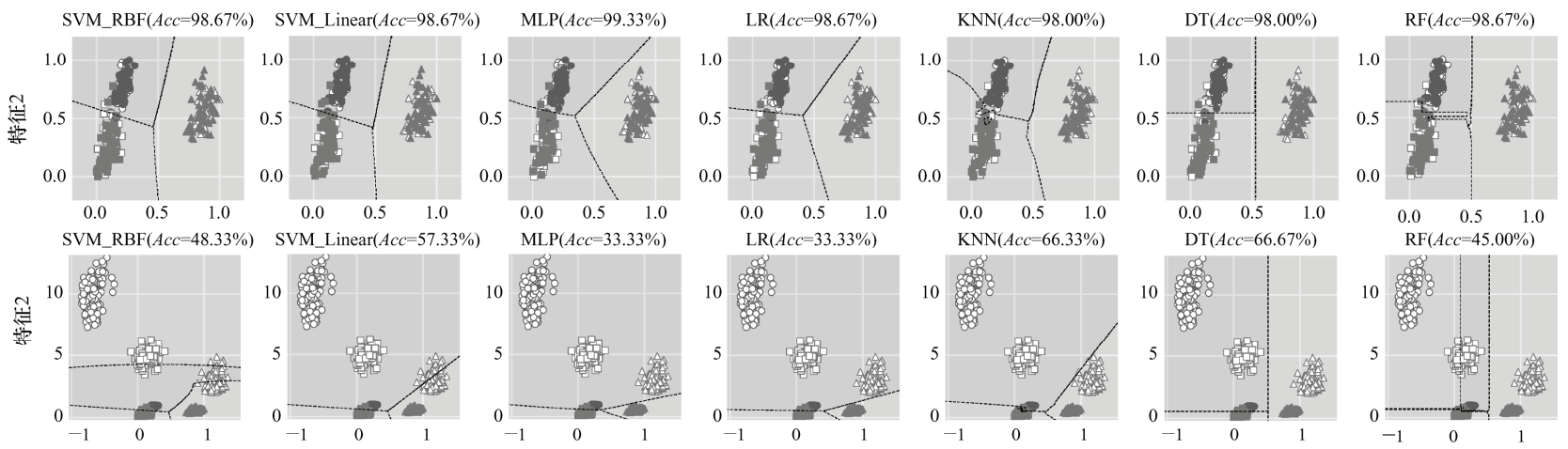

特征1

特征 1

特征 1

- 齿缺口-训练集

A 缺齿-训练集

○正常-训练集

特征 1

特征1

口齿缺口-测试集

$\Delta$ 缺齿-测试集

图 8 各故障识别方法在 30L-30L 和 30L-45H 两个任务中的二维决策面

滚动轴承任务_2D决策边界：CWRU_B $\rightarrow$ R2F_K任务

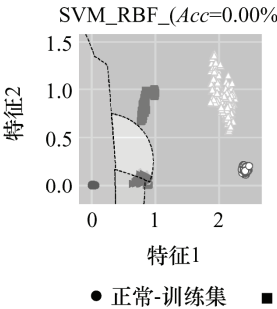

- 正常-训练集
MLP $(A c c=11.25 \%)$

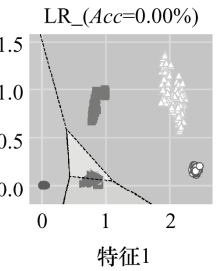

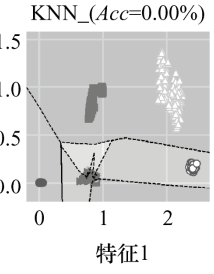

DT_ $(A c c=0.00 \%)$

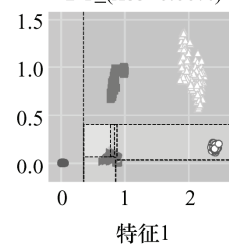

$\mathrm{RF}(A c c=0.00 \%)$

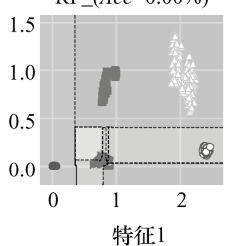

图 9 各故障识别方法在 CWRU_B-R2F_K 任务中的二维决策面

从这些结果中可以发现, 模型的复杂度不是影响 跨数据集诊断泛化性能的主要因素。不同来源数据集 间的同类样本在特征空间内的位置差异才是导致数 据驱动故障识别方法泛化性能下降的最直接原因。

(2) 对分布差异的分析。

接下来重点分析分布差异的影响, 我们将展示 各个方法的诊断性能与数据集间的分布差异有非常 强的关联性。利用 2.3 节 Eq.(4) 定义的统计参量 $D_{m m d}$ 对齿轮和滚动轴承各个诊断任务中的训练集和测试 集进行分布差异的度量, 所得到的结果展示在图 10 中。从图中可以发现以下几点。

1) 数据集间的分布距离矩阵与故障识别方法

齿轮数据集-分布距离度量 $\left(D_{m m d}\right)$

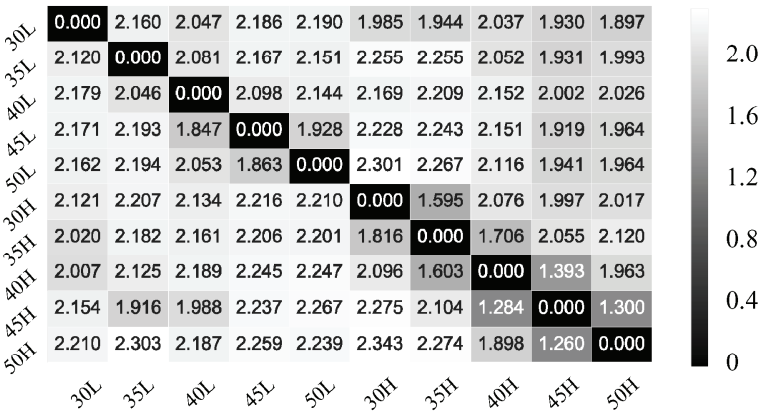

的识别精度矩阵相互对应(见图 6 和图 7), 分布距离 越小对应的识别精度越高, 反之则精度越低。

2) 从轴承识别任务中发现，设备差异对分布的 影响要大于工况差异所带来的影响。

3）分布距离矩阵并不对称, 这是由于基于不同 数据集所选择的 “最优” 特征集合存在差异。

通过上面的分析发现数据集间的分布差异 是导致跨数据集情形下诊断模型泛化性能下降 的主要因素。这意味着, 在这种诊断情形下设法 减小训练和测试集间的分布差异可能要比构建 新的不同复杂度的模式识别算法更加能够提升 诊断的精度。

滚动轴承数据集-分布距离度量 $\left(D_{m m d}\right)$

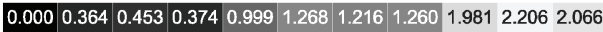
$\begin{array}{lllllllllll}0.301 & 0.000 & 0.278 & 0.251 & 0.935 & 1.125 & 1.051 & 1.021 & 1.898 & 1.875 & 1.812\end{array}$ $\begin{array}{lllllllllll}0.495 & 0.360 & 0.000 & 0.329 & 1.049 & 1.192 & 1.161 & 1.107 & 1.888 & 1.881 & 1.799\end{array}$ $\begin{array}{lllllllllllll}0.497 & 0.366 & 0.294 & 0.000 & 1.039 & 1.270 & 1.243 & 1.268 & 1.691 & 2.063 & 1.928\end{array}$ $\begin{array}{llllllllllll}0.826 & 0.886 & 0.943 & 0.918 & 0.000 & 0.526 & 0.538 & 0.636 & 1.814 & 1.952 & 1.866\end{array}$ $\begin{array}{lllllllllll}1.146 & 1.060 & 1.088 & 1.054 & 0.661 & 0.000 & 0.245 & 0.386 & 2.047 & 2.214 & 2.041\end{array}$ 1.0361 .0101 .0550 .9850 .5400 .2100 .0000 .2962 .0402 .2242 .060 $\begin{array}{lllllllllll}1.140 & 1.155 & 1.136 & 1.088 & 0.559 & 0.423 & 0.425 & 0.000 & 1.967 & 2.182 & 1.962\end{array}$

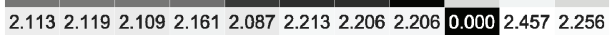

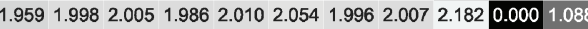

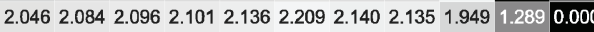

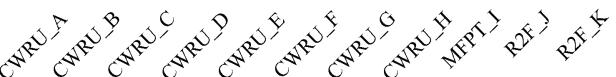

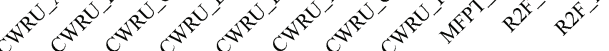




\section{2 对两个影响原因的进一步分析}

上述分析是对训练集和测试集在多维特征空间 内分布特性差异的整体度量。而一个特征空间是由 多个特征参量组成的, 每一个特征参量受到的影响 最终导致了两个数据集在整体特征空间内存在差 异。因此, 通过对单特征分布所受影响的分析可以 进一步从信号特性的角度探讨和解释导致跨数据集 诊断泛化性能下降的深层原因。

\subsection{1 可能的影响原因}

在机械设备的故障诊断问题中, 可能存在下述 影响数据集分布的原因。根据本文中所用数据的情 况, 将这些原因分为: 显在原因和潜在原因。显在 原因是所用数据间明确展示的差异, 包括: (1) 工况 (载荷或转速)差异; (2) 设备型号差异。潜在原因是 试验中可能存在的隐含影响, 它们包括: (1) 安装质 量差异; (2) 试验环境差异; (3) 故障程度差异; (4) 数 据采集方式差异等。

在下面的部分中重点分析两个显在原因对各个 特征参量产生的影响, 并从信号特性的角度进行了 进一步的解释。

\subsection{2 单特征分析的流程}

为了定量化分析各个特征受某一原因的影响, 在单一差异的识别任务中对各个特征参量进行分布 差异的分析。具体的分析流程如图 11 所示。首先, 在各个单特征 $\mathrm{F}_{i}$ 构成的一维空间内度量各个任务中 训练集和测试集的分布差异, 形成近似图 10 的特征 $\mathrm{F}_{i}$ 分布差异度量矩阵 (但是为对称矩阵); 然后, 在该 矩阵中选择只有单一影响因素变化的所有任务对,

\section{输入端信号特征}

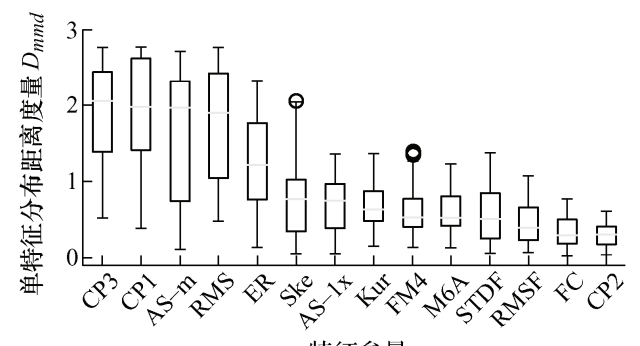

特征参量

(a) 齿轮诊断任务

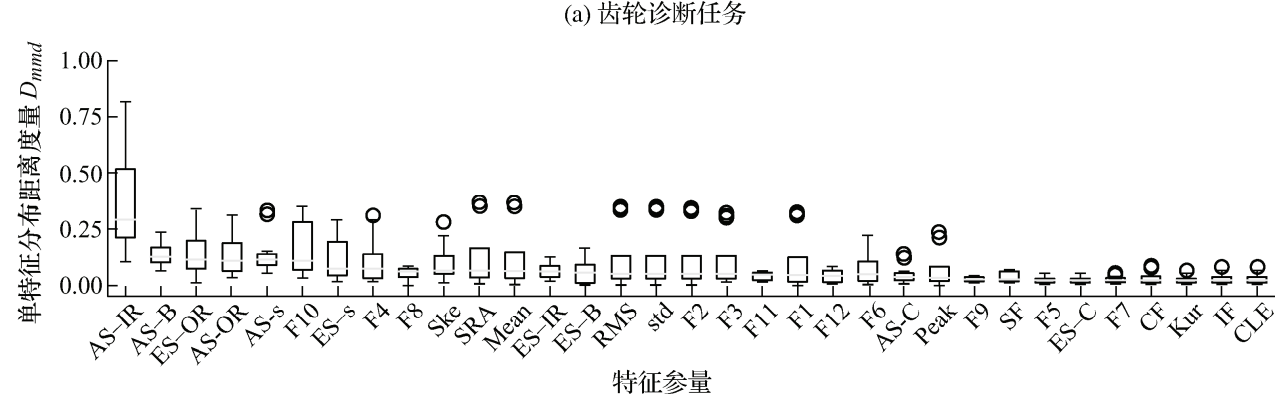

(b) 滚动轴承诊断任务

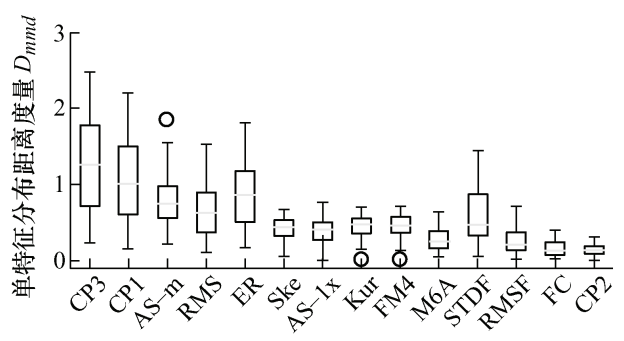

例如轴承诊断中只有工况变化时将选择 $\mathrm{A}-\mathrm{B} / \mathrm{A}-$ C/A-D/B-C/B-D/C-D 以及 E-F/E-G/E-H/F-G/ F-H/G-H 任务。最后, 将这些任务间的单特征分布差异值以 箱型图(Box-plot)的形式展示, 通过中位数大小对各 个 Box 进行排序, 从而比较各个特征在单一因素下 所受到的影响大小。

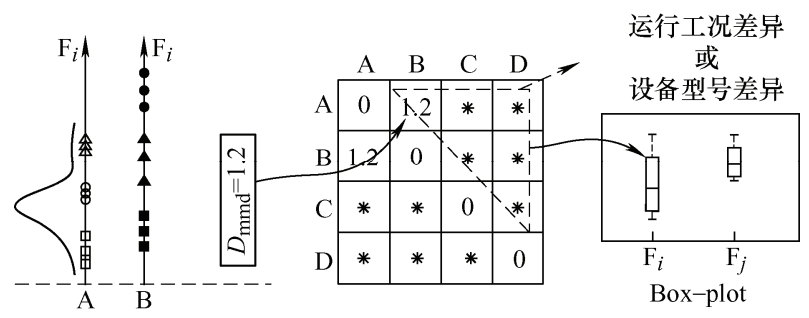

图 11 单个特征所受影响分析的流程

\subsection{3 分析结果和讨论}

工况差异下齿轮和轴承两个任务中各个特征所 受的影响展示在图 12 中。只有在轴承任务中才存在 设备型号差异因素的影响情形, 轴承各个特征在该 差异下的影响展示在图 13 中。通过这些结果可以发 现下面几个现象。

(1) 对于轴承诊断任务, 通过对比图 12 和图 13 的 $D_{m m d}$ 值大小发现设备型号差异对每一个特征分 布偏移所带来的影响要大于工况差异所带来的影响 (对应解释第 3.3 节的(3)现象)。

(2) 对于工况差异情形(图 12), 无论是齿轮任务 还是轴承任务, 频率中心 $\mathrm{FC}$ (轴承任务中为 F5) 以及 近似含义的特征(RMSF/STDF/CP2, F7/F8/F9)所受 影响相对其他特征而言较小。

输出端信号特征

特征参量

图 12 工况差异因素下齿轮和轴承诊断任务各个特征所受影响对比 


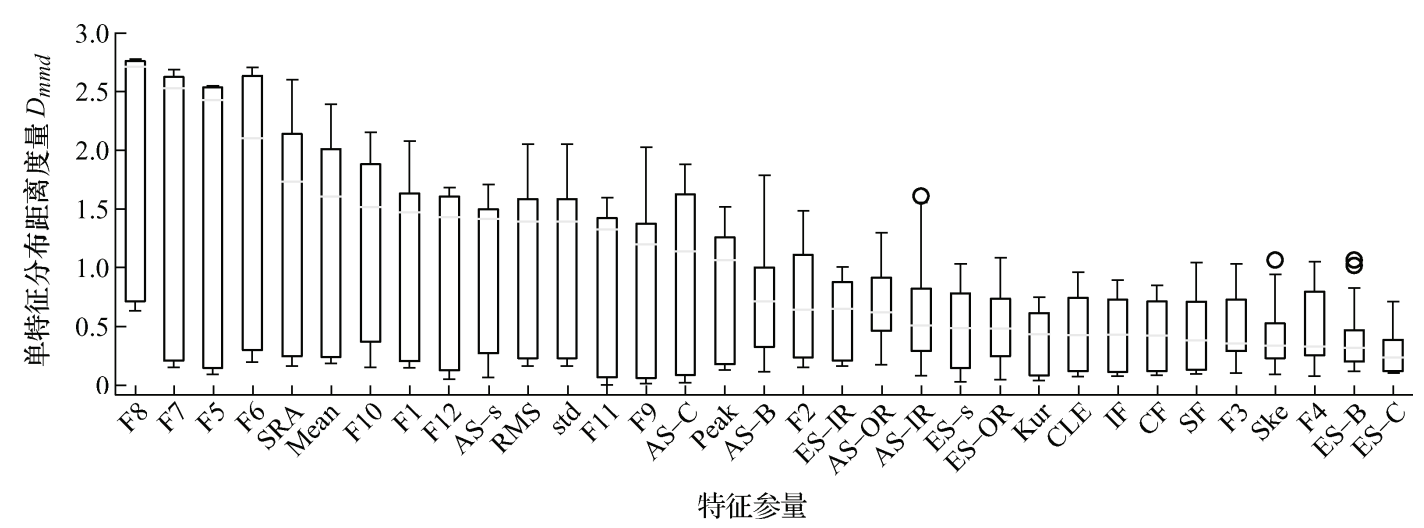

图 13 型号差异因素下轴承诊断任务各个特征所受影响对比

(3) 从图 12 的轴承诊断任务中可见, 在工况差 异情形下无量纲统计参量 SF/CF/IF/CLE, 以及 Kur 所受影响也相对较小。

(4) 对于轴承诊断任务, 在型号差异情形下 (图 13)频率中心 F5 以及近似含义的特征 F7/F8 所受 影响最大; 而无量纲统计参量 $\mathrm{SF} / \mathrm{CF} / \mathrm{IF} / \mathrm{CLE}$ 以及 Ske 所受影响相对很小。

通过上面的分析可以明确工况差异和设备型号 差异主要影响了哪些特征参量, 从而导致了不同数 据集间在特征空间内分布不同, 进而影响泛化性能。 接下来进一步从信号表现特性的角度分析它们导致 特征分布差异的深层次原因。

\subsection{4 信号特性角度的解释}

该部分主要从信号特性的角度对上述两个 现象进行进一步解释: (1) 为何频率中心类的特 征在工况差异时所受影响最小, 而在设备型号差 异时所受影响最大? (2) Kur 和 Ske 指标受不同因 素影响的进一步解释。主要对滚动轴承任务进行 分析。

(1) 对频率中心 FC 的分析。

频率中心 $\mathrm{FC}=\left[\sum_{i=1}^{K} f_{i} \mathcal{F}_{i}\right] /\left[\sum_{i=1}^{K} \mathcal{F}_{i}\right]$ 度量的 是频率谱线以其幅值为权重的加权平均, 描述频谱 中主要频率成分所处的位置。在轴承任务工况变化 情形中, $\mathrm{A} / \mathrm{B} / \mathrm{C} / \mathrm{D}$ 和 $\mathrm{E} / \mathrm{F} / \mathrm{G} / \mathrm{H}$ 数据集各个健康状态 振动信号的幅值谱和频率中心值(虚线)分别展示在 图 14a、14b 中。在轴承任务设备型号差异情形中, $\mathrm{A} / \mathrm{E} / \mathrm{I} / \mathrm{K}$ 数据集相同模式的振动信号频率和频率中 心值展示在图 15 中。

那么, 是什么导致工况变化时对频率中心的影 响很小, 而在设备差异时对其影响很大呢? 第一, 轴承故障时会产生宽频的冲击信号, 从而激励起高 频的振动 ${ }^{[39]}$, 这个现象可以通过横向比较图 14a 中 正常和故障状态的振动信号频谱得到证实。第二, 这个高频的振动与轴承以及轴承所工作的设备的固
有振动频率有关, 甚至还与轴承的安装, 比如预紧 力等有关, 但是它与设备的运行工况无关, 这可以 通过纵向对比图 14a 和图 $14 \mathrm{~b}$ 中同一状态不同数据 集的频谱得到证实, 这是频率中心在工况差异下所 受影响较小的原因。第三, 由于不同轴承(以及轴承 工作的设备)的固有频率不同, 因此在设备差异下频 率中心所受的影响较大, 这个现象可以通过纵向对 比图 15 中同一状态不同数据集的频谱得到证实。上 述分析说明了频率中心及与其有相近含义的特征在 工况差异时所受影响小, 设备差异时所受影响大的 原因。

(2) 对峭度和偏度的分析。

该研究中所利用的峭度 $\operatorname{Kur}=\left[\sum_{i=1}^{N}\left(x_{i}-\bar{x}\right)^{4}\right] /$ $\left[(N-1) \sigma_{1}^{4}\right]$ 和 偏度 $\mathrm{Ske}=\left[\sum_{i=1}^{N}\left(x_{i}-\bar{x}\right)^{3}\right] /\left[(N-1) \sigma_{1}^{3}\right]$ 在文献[44]中被称为峭度系数和偏度系数。Kur 反映 的是数据分布形态偏离正太分布的分散或集中程 度, 能够用来衡量振动信号中的冲击特性; Ske 反 映的是振动信号分布的对称形态, 当旋转机械存在 某一固定方向的摩擦或碰撞时, 可能导致偏度值变 大。为了分析不同数据集下振动信号的分布形态, 这里采用核密度估计方法来估计振动信号的概率密 度函数 ${ }^{[45]}$ 。图 16 展示了 $\mathrm{A} / \mathrm{B} / \mathrm{C} / \mathrm{D}$ 四个数据集中同 类型状态下振动信号的概率密度函数和相应的 Kur 和 Ske 值, 可以发现当工况发生差异时同一状态 下的 Kur 发生很小的变化, 并且概率密度函数基 本是对称的偏度值的变化也很小, 因此在工况差 异情形下 Kur 和 Ske 所受影响较小。图 17 中展示 了设备差异情形下振动信号的概率密度函数, 可 以发现对于不同的设备而言, 当发生故障时 Kur 虽然均增加(表明产生了冲击), 但是增加的幅度相 差较大; 此外, 振动信号同样呈现对称的分布, Ske 值相差不大。因此 Ske 值在设备差异下受到的 影响要小于 Kur 值。 

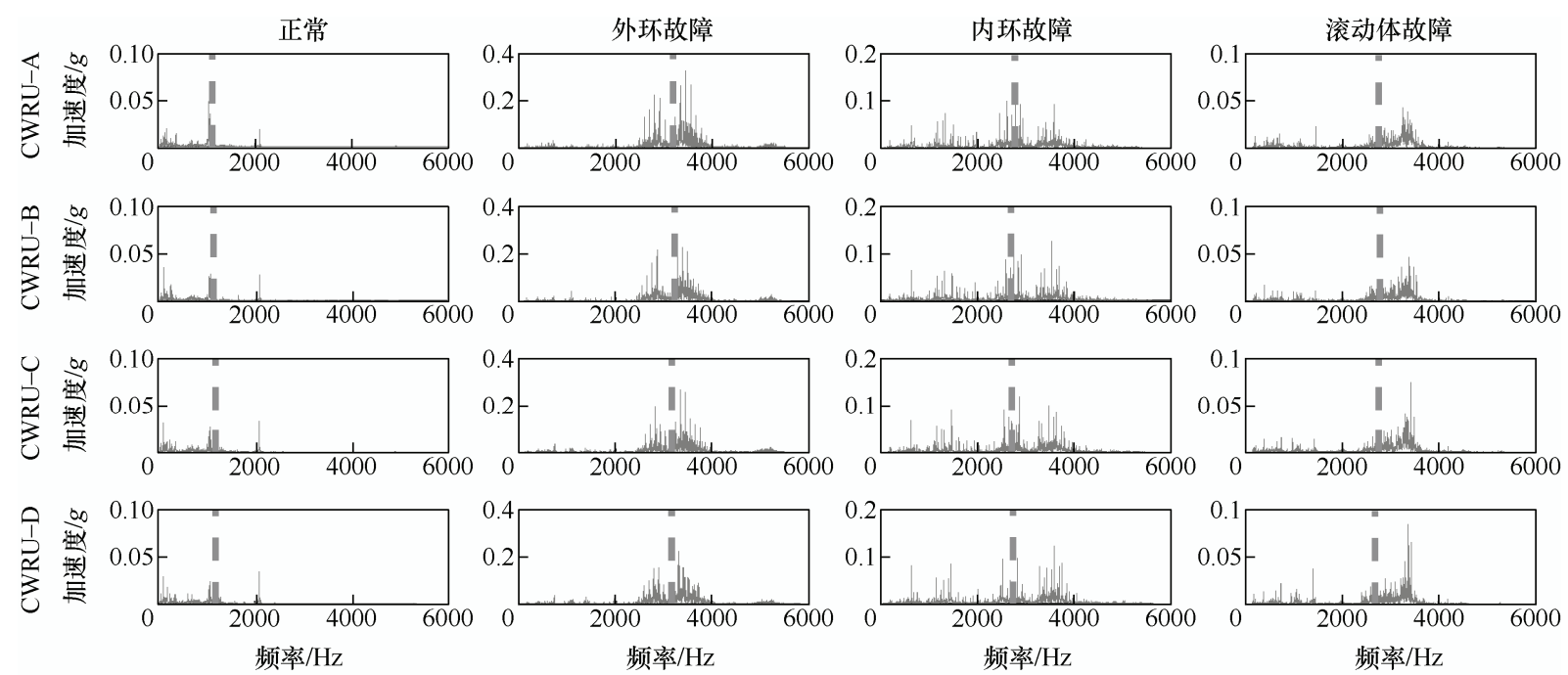

(a) A-D 数据集间的对比
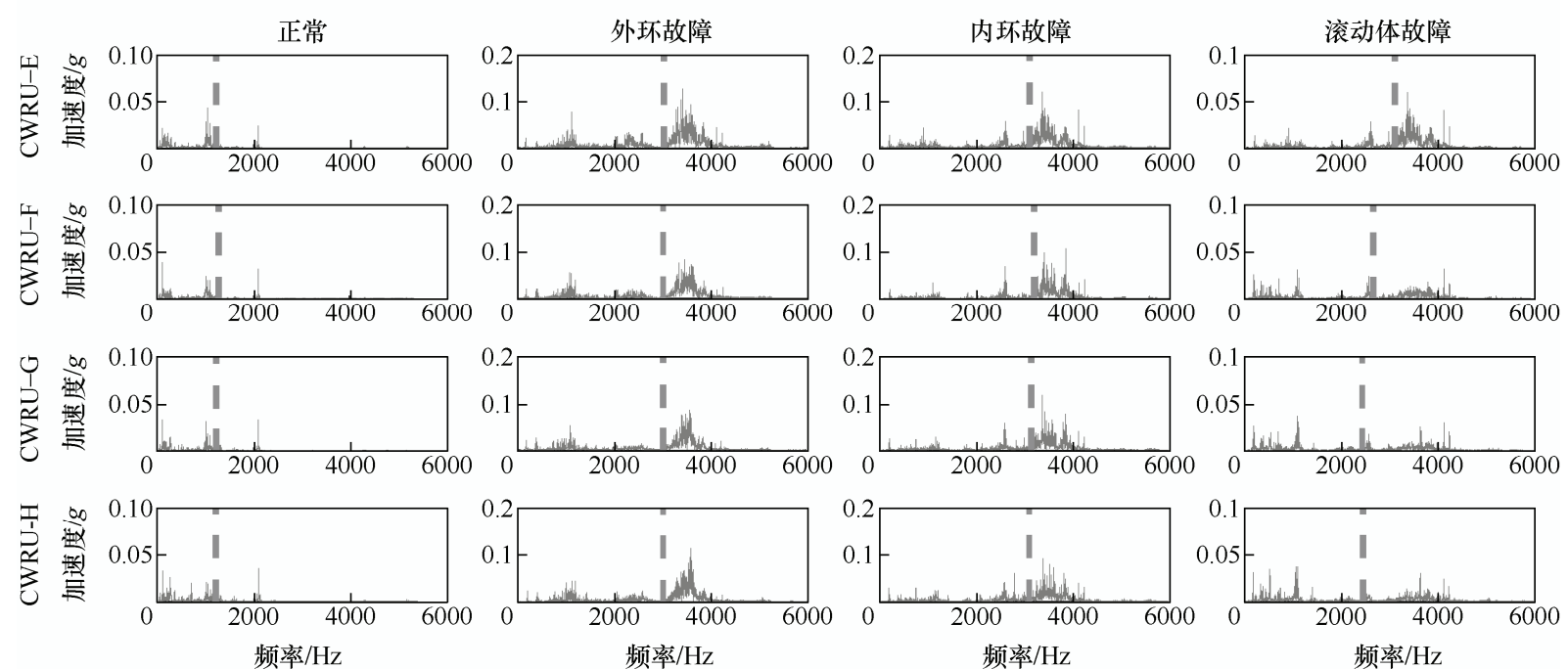

(b) E-H 数据集间的对比

图 14 工况差异下滚动轴承各数据集的振动信号频谱和频率中心 F5 对比
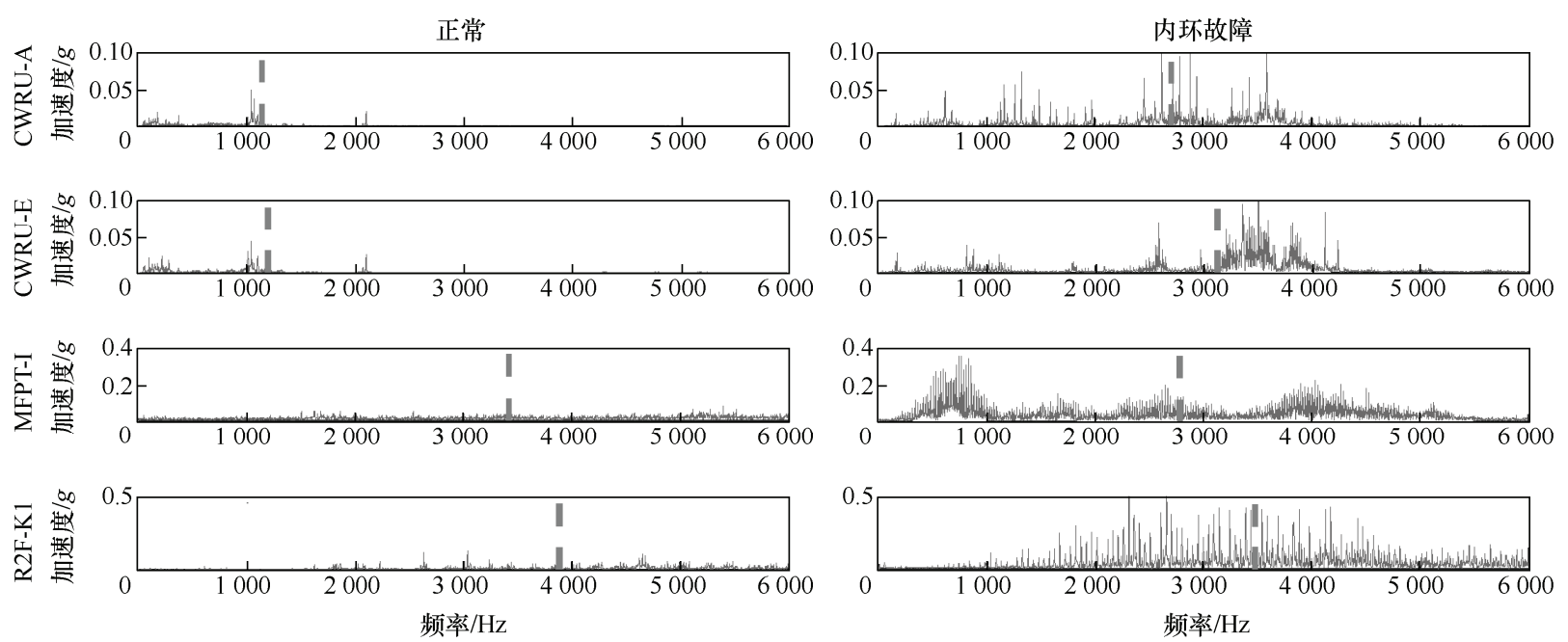

图 $15 \mathrm{~A} / \mathrm{E} / \mathrm{I} / \mathrm{K}$ 数据集正常和内环故障状态振动信号的频谱和频率中心 F5 对比 
正常状态信号

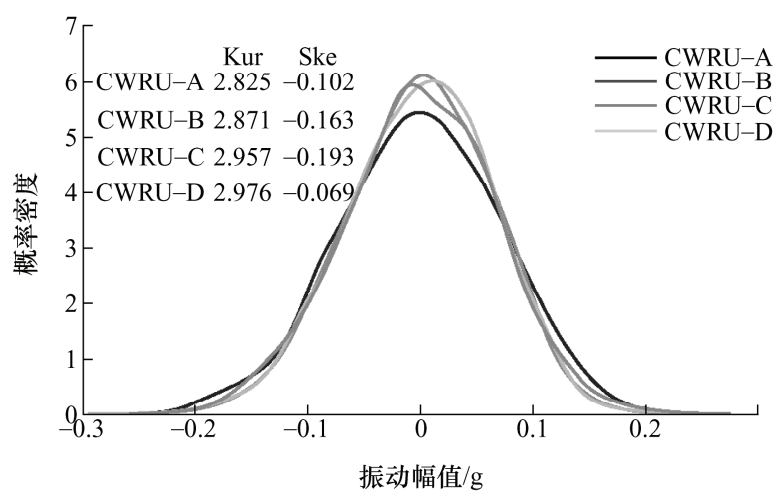

内环故障信号

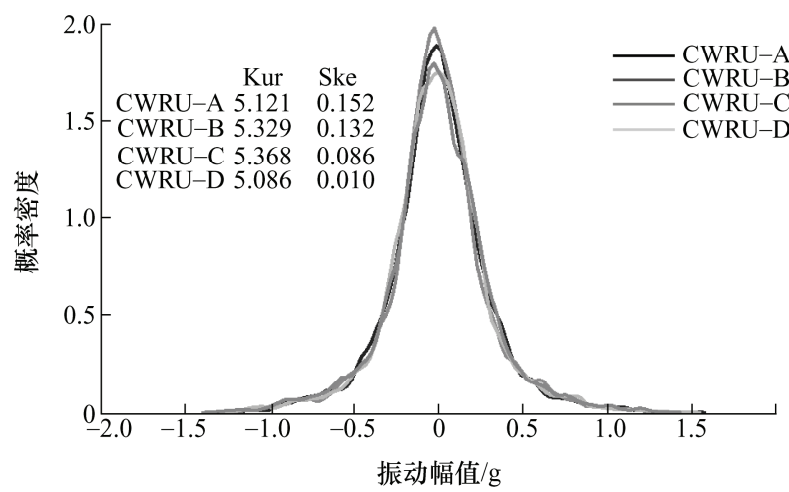

外环故障信号

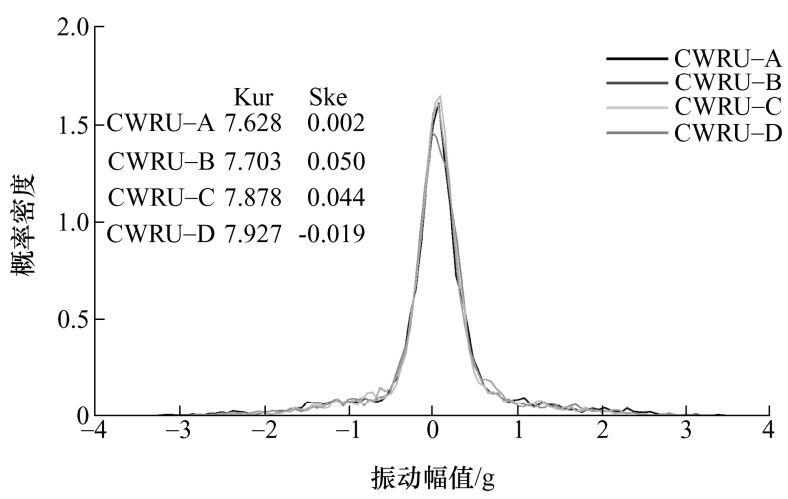

滚动体故障信号

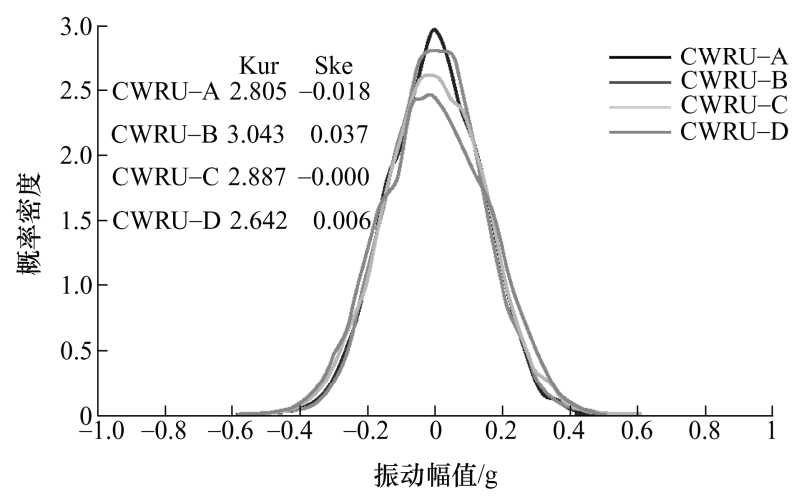

图 $16 \mathrm{~A} / \mathrm{B} / \mathrm{C} / \mathrm{D}$ 数据集各状态振动信号概率密度函数以及 Kur 和 Ske 值对比

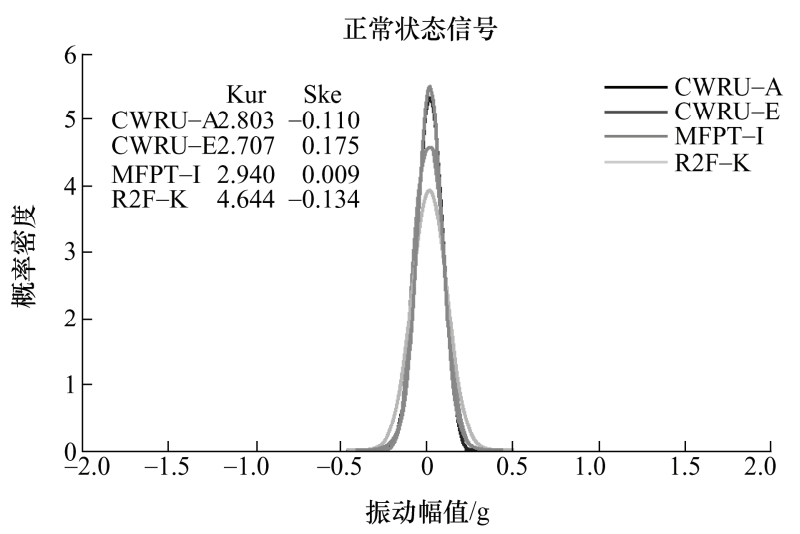

内环故障信号

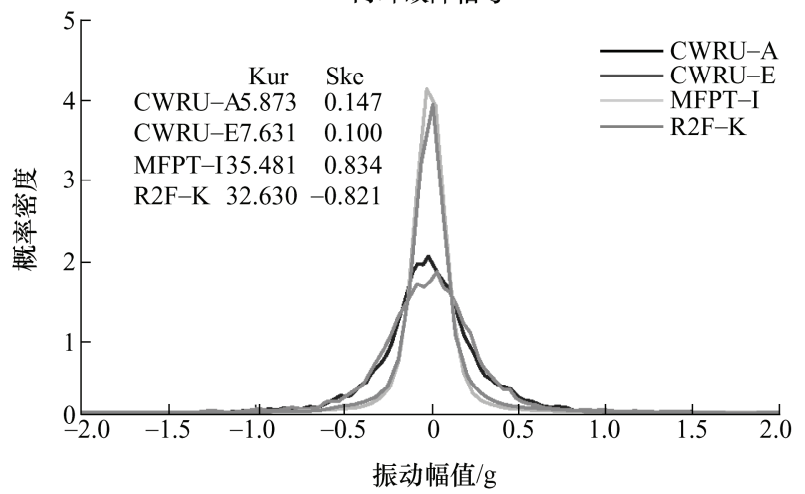

图 $17 \mathrm{~A} / \mathrm{E} / \mathrm{I} / \mathrm{K}$ 数据集正常和内环故障状态振动信号概率密度函数以及 Kur 和 Ske 值对比

\section{5 结论}

在本文中, 首先讨论了数据驱动故障诊断方法 面对实际诊断问题的数据困境, 进而从获取数据可 行性的角度出发提出利用来自同设备但不同工况、 同型号但不同设备或其他同类型设备的累积故障数 据构建识别模型的跨数据集诊断问题。通过构建多 个齿轮和滚动轴承数据集, 发现多个数据驱动诊断 方法在跨数据集诊断情形下的泛化性能均会降低, 尽管各个方法在同一数据集的诊断问题中均有较高 的识别精度。通过对比分析各个诊断任务下训练集
和测试集的分布差异，发现两个数据集间的分布差 异是导致诊断方法泛化性能下降的主要因素。进一 步的分析主要集中在单一特征所受影响的分析上, 主要从信号特性分析的角度解释了工况差异和设备 型号差异两个因素是如何影响分布差异的。其中发 现频率中心类特征参量在工况差异下受到的影响很 小, 但是在设备差异时所受的影响很大, 通过对比 分析不同设备的诊断信号频谱发现设备的固有振动 频率差异可能是导致这个现象的主要原因。此外, 发现 Kur 和 Ske 参数在工况差异下受的影响很小, 并通过估计振动信号的概率密度函数进一步解释了 该现象。本文的研究对于构建面向实际诊断情形的 
有效跨域故障诊断方法具有积极的指导意义。

本研究存在下述的一些限制和不足。

(1) 由于可用数据的限制, 只讨论了工况差异 和设备型号差异所造成的影响, 并且这其中可能存 在其他潜在因素的影响。

(2) 对于齿轮以及滚动轴承的故障诊断, 除了 本研究中利用到的特征参量, 还有许多其他先进的 故障特征提取方法 ${ }^{[6-9]}$, 这些特征在该研究中没有考 虑, 它们所受影响性大小以及与文中所用特征的对 比结果没有被分析。

(3) 本文中只是讨论了特征有利于泛化的一个 方面特性, 即受工况、设备等因素的影响。此外一 个有利于泛化的特征同时应该具有稳定一致区分不 同故障模式的能力, 这个特性在本文中没有被讨论。

在未来的研究中, 我们将重点关注如何解决跨 数据集诊断的问题, 拟从特征层面研究提取更加稳 定的一致性特征, 以及从识别模型层面研究迁移诊 断知识来构建更加有泛化能力的识别模型。

\section{参 考 文 献}

[1] LEE J, WU Fangji, ZHAO Wenyu, et al. Prognostics and health management design for rotary machinery systemsreviews, methodology and applications[J]. Mechanical Systems and Signal Processing, 2014, 42(1-2): 314-334.

[2] YIN Shen, LI Xianwei, GAO Huijun, et al. Data-based techniques focused on modern industry: An overview[J]. IEEE Transactions on Industrial Electronics, 2015, 62(1): 657-667.

[3] 王国彪, 何正嘉, 陈雪峰, 等. 机械故障诊断基础研究 “何去何从” [J]. 机械工程学报, 2013, 49(1): 63-72. WANG Guobiao, HE Zhengjia, CHEN Xuefeng, et al. Basic research on machinery fault diagnosis - what is the prescription[J]. Journal of Mechanical Engineering, 2013, 49(1): 63-72.

[4] 雷亚国, 贾峰, 孔德同, 等. 大数据下机械智能故障诊 断的机遇与挑战[J]. 机械工程学报, 2018, 54(5): 94-104. LEI Yaguo, JIA Feng, KONG Detong, et al. Opportunities and challenges of machinery intelligent fault diagnosis in big data era[J]. Journal of Mechanical Engineering, 2018, 54(5): 94-104.

[5] CHEN Xuefeng, WANG Shibin, QIAO Baijie, et al. Basic research on machinery fault diagnostics: Past, present, and future trends[J]. Frontiers of Mechanical Engineering, 2018, 13(2): 264-291.

[6] FENG Zhipeng, LIANG Ming, CHU Fulei. Recent advances in time - frequency analysis methods for machinery fault diagnosis: a review with application examples[J]. Mechanical Systems and Signal Processing, 2013, 38(1): 165-205.

[7] LEI Yaguo, LIN Jing, HE Zhengjia, et al. A review on empirical mode decomposition in fault diagnosis of rotating machinery[J]. Mechanical Systems and Signal Processing, 2013, 35(1-2): 108-126.

[8] LI Yongbo, WANG Xianzhi, LIU Zhenbao, et al. The entropy algorithm and its variants in the fault diagnosis of rotating machinery: a review[J]. IEEE Access, 2018, 6: 66723-66741.

[9] WANG Yanxue, XIANG Jiawei, MARKERT R, et al. Spectral kurtosis for fault detection, diagnosis and prognostics of rotating machines: a review with applications[J]. Mechanical Systems and Signal Processing, 2016, 66-67: 679-698.

[10] BIET M. Rotor faults diagnosis using feature selection and nearest neighbors rule: Application to a turbogenerator[J]. IEEE Transactions on Industrial Electronics, 2013, 60(9): 4063-4073.

[11] ILlias H A, CHAI Xinrui, ABU BAKAR A H. Hybrid modified evolutionary particle swarm optimisation-time varying acceleration coefficient-artificial neural network for power transformer fault diagnosis[J]. Measurement, 2016, 90: 94-102.

[12] ZHANG Xiaoli, CHEN Wei, WANG Baojian, et al. Intelligent fault diagnosis of rotating machinery using support vector machine with ant colony algorithm for synchronous feature selection and parameter optimization[J]. Neurocomputing, 2015, 167: 260-279.

[13] LEI Yaguo, JIA Feng, LIN Jing, et al. An intelligent fault diagnosis method using unsupervised feature learning towards mechanical big data[J]. IEEE Transactions on Industrial Electronics, 2016, 63(5): 3137-3147.

[14] KHAN S, YAIRI T. A review on the application of deep learning in system health management[J]. Mechanical Systems and Signal Processing, 2018, 107: 241-265.

[15] ZHAO Rui, YAN Ruqiang, CHEN Zhenghua, et al. Deep learning and its applications to machine health monitoring $[\mathrm{J}]$. Mechanical Systems and Signal Processing, 2019, 115: 213-237.

[16] LEI Yaguo, ZUO M J, HE Zhengiia, et al. A multidimensional hybrid intelligent method for gear fault diagnosis[J]. Expert Systems with Applications, 2010, 
37(2): 1419-1430.

[17] JIA Feng, LEI Yaguo, LIN Jing, et al. Deep neural networks: A promising tool for fault characteristic mining and intelligent diagnosis of rotating machinery with massive data[J]. Mechanical Systems and Signal Processing, 2016, 72-73: 303-315.

[18] LI Yongbo, YANG Yuantao, WANG Xianzhi, et al. Early fault diagnosis of rolling bearings based on hierarchical symbol dynamic entropy and binary tree support vector machine[J]. Journal of Sound and Vibration, 2018，428: 72-86.

[19] ZHAO Rui, WANG Dongzhe, YAN Ruqiang, et al. Machine health monitoring using local geature-based gated recurrent unit networks[J]. IEEE Transactions on Industrial Electronics， 2018， 65(2): 1539-1548.

[20] SHAO Haidong, JIANG Hongkai, LIN Ying, et al. A novel method for intelligent fault diagnosis of rolling bearings using ensemble deep auto-encoders[J]. Mechanical Systems and Signal Processing, 2018, 102: 278-297.

[21] BEN-DAVID S, BLITZER J, CRAMMER K, et al. Analysis of representations for domain adaptation[C]/Advances in Neural Information Processing Systems, December 04-07, 2006, Canada. Massachusetts : MIT Press Cambridge, 2007: 137-144.

[22] SU Zuqiang, TANG Baoping, MA Jinghua, et al. Fault diagnosis method based on incremental enhanced supervised locally linear embedding and adaptive nearest neighbor classifier[J]. Measurement, 2014, 48: 136-148.

[23] JIA Feng, LEI Yaguo, GUO Liang, et al. A neural network constructed by deep learning technique and its application to intelligent fault diagnosis of machines[J]. Neurocomputing, 2018, 272: 619-628.

[24] LI Yongbo, YANG Yuantao, LI Guoyan, et al. A fault diagnosis scheme for planetary gearboxes using modified multi-scale symbolic dynamic entropy and mRMR feature selection[J]. Mechanical Systems and Signal Processing, 2017, 91: 295-312.

[25] YANG Bo Suk, XIAO Di, TIAN Han. Random forests classifier for machine fault diagnosis[J]. Journal of Mechanical Science and Technology, 2008, 22(9): 1716-1725.

[26] WANG Ziwei, ZHANG Qinghua, XIONG Jianbin, et al. Fault diagnosis of a rolling bearing using wavelet packet denoising and random forests[J]. IEEE Sensors Journal, 2017, 17(17): 5581-5588.
[27] KUAI Moshen, CHENG Gang, PANG Yusong, et al. Research of planetary gear fault diagnosis based on permutation entropy of CEEMDAN and ANFIS[J]. Sensors, 2018, 18(3): 782.

[28] LEI Yaguo, HE Zhengiia, ZI Yanyang. A new approach to intelligent fault diagnosis of rotating machinery[J]. Expert Systems with Applications, 2008, 35(4) : 1593-1600.

[29] VAPNIK V. The nature of statistical learning theory[M]. New York: Springer, 2000.

[30] TAN P, STEINBACH M, KARPATNE A, et al. Introduction to data mining[M]. 2nd ed. Singapore: Pearson Education, 2011.

[31] 周志华. 机器学习 $[\mathrm{M}]$. 北京: 清华大学出版社, 2016 . ZHOU Zhihua. Machine learning[M]. Beijing: Tsinghua University Press, 2016.

[32] VACHTSEVANOS G, LEWIS F L, ROEMER M, et al. Intelligent fault diagnosis and prognosis for engineering systems: methods and case studies[M]. Wiley Online, 2006.

[33] PHM Society. PHM 09 data challenge data[EB/OL]. [2018-11-07]. https://www.phmsociety.org/competition/ PHM/09/apparatus.

[34] LOPARO K. Case Western Reserve University Bearing Data Center [EB/OL]. [2018-11-07]. http://csegroups. case.edu/bearingdatacenter/pages/download-data-file.

[35] BECHHOEFER E. Society for Machinery Failure Prevention Technology. [EB/OL]. [2018-11-07]. https://mfpt. org/fault-data-sets/.

[36] SHARMA V, PAREY A. A review of gear fault diagnosis using various condition indicators[J]. Procedia Engineering, 2016, 144: 253-263.

[37] CERRADA M, ZURITA G, CABRERA D, et al. Fault diagnosis in spur gears based on genetic algorithm and random forest $[\mathrm{J}]$. Mechanical Systems and Signal Processing, 2016, 70-71: 87-103.

[38] LEI Yaguo, HE Zhengjia, ZI Yanyang. Application of an intelligent classification method to mechanical fault diagnosis[J]. Expert Systems with Applications, 2009, 36(6): 9941-9948.

[39] RANDALL R, ANTONI J. Rolling element bearing diagnostics-a tutorial[J]. Mechanical Systems and Signal Processing, 2011, 25(2): 485-520.

[40] GRETTON A, BORGWARDT K M, RASCH M, et al. A kernel two-sample test[J]. Journal of Machine Learning 
Research, 2012, 13: 723-773.

[41] 李航. 统计学习方法[M]. 北京: 清华大学出版社, 2012. LI Hang. Statistical learning method[M]. Beijing : Tsinghua University Press, 2012.

[42] BREIMAN L. Random forests[J]. Machine Learning, 2001, 45(1): 5-32.

[43] PENG Hanchuan, LONG Fuhui, DING C. Feature selection based on mutual information criteria of maxdependency, max-relevance, and min-redundancy[J]. IEEE Transactions on Pattern Analysis and Machine Intelligence, 2005, 27(8): 1226-1238.

[44] 钟秉林, 黄仁. 机械故障诊断学 [M]. 3 版. 北京: 机械
工业出版社， 2006.

ZHONG Binglin, HUANG Ren. Introduction to machine fault diagnosis[M]. 3rd ed. Beijing: China Machine Press, 2006.

[45] CAO R, CUEVAS A, MANTEIGA W. A comparative study of several smoothing methods in density estimation[J]. Computational Statistics \& Data Analysis, 1994, 17(2): 153-176.

作者简介: 郑怀亮, 男, 1989 年出生, 博士研究生。主要研究方向为机 械设备的故障诊断, 智能诊断算法和迁移学习。

E-mail: hlzhenghit@126.com

王日新(通信作者), 男, 1963 年出生, 博士。副教授。主要研究方向为 航天器和机械设备智能诊断技术。

E-mail: wangrx@hit.edu.cn 